\title{
Blob Stability and Transport in the Scrape-off-layer
}

\author{
D. A. D'Ippolito and J. R. Myra \\ Lodestar Research Corporation, Boulder, Colorado, USA
}

May 23, 2003

(submitted to Physics of Plasmas) 


\title{
Blob Stability and Transport in the Scrape-off-layer *
}

\author{
D. A. D'Ippolito ${ }^{\dagger}$ and J. R. Myra \\ Lodestar Research Corporation, 2400 Central Avenue, Boulder, Colorado 80301
}

\begin{abstract}
High-density plasma filaments extended along the magnetic field, which look like "blobs" in the plane perpendicular to $\mathbf{B}$, have been observed in the scrape-off-layer (SOL) of many plasma devices. These objects become polarized and subsequently $\mathbf{E} \times \mathbf{B}$ drift to the wall carrying a significant flux of particles. This mechanism may account for the observed nondiffusive, intermittent transport in the far SOL of tokamaks. Previous work has examined simple models of blob propagation and shown that the radial convective velocity $\mathrm{u}_{\mathrm{x}}$ has the scaling $\mathrm{u}_{\mathrm{x}} \propto \mathrm{y}_{\mathrm{b}}{ }^{-2}$, where $\mathrm{y}_{\mathrm{b}}$ is the poloidal blob dimension. Thus, the radial particle flux is sensitive to the details of the blob size and shape distributions; these in turn are affected by various secondary instabilities which cause blob fragmentation. A simple model of blob instability (driven by curvature in the presence of sheath boundary conditions) is studied by $1 \mathrm{D}$ calculations and 2D simulations to provide insight on the stability and dynamics of blobs. We show that a competition between the time scales for instability and transport determines the range of allowed blob sizes and shapes and constrains the overall radial velocity.
\end{abstract}

PACS numbers: 52.25.Fi, 52.35.Ra, 52.55.Dy, 52.55.Fa

†email: dasd@lodestar.com 


\section{Introduction}

There is growing evidence that a significant fraction of the radial particle transport in the scrape-off-layer (SOL) is non-diffusive and intermittent in space and time. ${ }^{1-13}$ This transport is convective in nature and extends to the far SOL, typically yielding flat density profiles and a significant particle flux near the wall.4,14,15 The increased recycling at the walls, as evidenced by the "main chamber recycling regime", 4 can result in reduced divertor efficiency and increased impurities. The convective transport is more important in discharges with higher density, and may be associated with the density limit. ${ }^{16}$ Thus, there is an increasing experimental and theoretical effort to understand the source of this convective transport.

Recently, a theoretical model was developed 17,18 that accounts for some of these properties. The model is consistent with the high-density plasma filaments observed in the SOL of NSTX. ${ }^{13,19}$ These filaments are spatially extended along the field line but are localized in the plane perpendicular to $\mathbf{B}$ and look like "blobs" in this plane. Density blobs have been seen with gas puff imaging diagnostics $8,11,13,19$ on several experiments and are observed to move in both the radial (x) and poloidal (y) directions. The density blob theory 17,18 treats the blobs as coherent structures which propagate outwards at the outboard midplane by a simple mechanism: the curvature drift (for tokamaks) or centrifugal force (for linear rotating machines) gives rise to a poloidal charge separation, induced poloidal electric field, and subsequent radial $\mathbf{E} \times \mathbf{B}$ drift.

In the simplest version of the model (constant temperature, neglect of vorticity, no background density), an assumed blob density of the form $n(x, y)=n(x) \exp \left(-y^{2} / 2 y_{b}^{2}\right)$ leads to a solution with a constant radial velocity $\mathrm{u}_{\mathrm{x}}$,

$$
u_{x}=\frac{\beta}{\alpha y_{b}^{2}}
$$

where $\beta \propto 1 / \mathrm{R}$ is the curvature parameter, and $\alpha \propto 1 / \mathrm{qR}$ is the parameter representing the parallel losses to the sheaths. The factor $\alpha$ in Eq. (1) results from the axial sheath boundary condition for $J_{\|}$in the vorticity equation. All quantities in Eq. (1) are 
dimensionless, using the normalizations of Ref.18 (see also Sec. II). There are two points to note in Eq. (1): (i) the blob transport arises from a balance between the curvature drift term and the sheath conductivity, ${ }^{20}$ as represented by the factor $\beta / \alpha$; and (ii) the radial velocity and radial particle flux $\Gamma=\mathrm{nu}_{\mathrm{x}}$ are functions of the poloidal blob size $\mathrm{y}_{\mathrm{b}}$, which is the distance over which the curvature-induced charge separation occurs.

The second point suggests that the radial transport from an ensemble of blobs depends sensitively on the distributions of blob size and shape. The dependence of the radial transport on the blob size can be seen by comparing the time $\tau_{\mathrm{c}}=\Delta \mathrm{x} / \mathrm{u}_{\mathrm{x}} \propto \mathrm{y}_{\mathrm{b}}^{2}$ to convect a radial distance $\Delta \mathrm{x}$ with the parallel flow time $\tau_{\|}=\mathrm{L}_{\|} / \mathrm{c}_{\mathrm{S}}$ for particle flow to the sheaths. Only blobs small enough that $\tau_{\mathrm{c}}<\tau_{\|}$will contribute to the radial transport; larger blobs will be depleted of particles before moving a significant distance. This same physics can be made more quantitative by averaging the convective particle flux over an ensemble of blob sizes, as discussed in Ref. 18.

Another time scale is introduced by stability considerations, which is the subject of the present paper. Secondary instabilities (driven by the blob internal pressure profile) can modify the blob size and shape distributions by breaking up large blobs into smaller ones. Since the smaller child blobs move faster than their parents, the net transport is increased by the instability. The interesting parameter regime is $\gamma^{-1} \leq \tau_{c} \ll \tau_{\|}$in which the maximum growth rate $\gamma$ is fast enough to break up the blob before it moves a significant distance outwards or loses any particles to the sheaths. Since $\gamma$ and $\tau_{\mathrm{c}}$ both depend on the blob size, the condition $\gamma \tau_{\mathrm{c}}=1$ (balancing stability and transport time scales) determines a cut-off on the maximum blob size in the ensemble. This characteristic scale length in turn sets a lower bound on the radial velocity expected for the blobs.

To avoid confusion in terminology, note that we use the term blob "size" to refer to its linear dimensions (or area) in the plane perpendicular to $\mathbf{B}$, not to be confused with the blob "amplitude" or "height," which is the difference between the peak blob density and the background density. In numerical simulations with a background density, it is found that the blob velocity $\mathrm{u}_{\mathrm{x}}$ also depends on the blob height, as discussed in Sec.IV. 
There are a number of experimental measurements ${ }^{5-13}$ and simulations ${ }^{21}$ suggesting that turbulent processes create large blobs (several cm across) in the SOL near the separatrix. These turbulent processes are driven by the "primary" instabilities of the SOL profiles near the separatrix, typically drift-resistive instabilities such as the RX mode. ${ }^{21,22}$ The effect of the birth processes on the size and shape distributions of the blobs is outside the scope of this paper. We restrict the present analysis to "secondary" instabilities driven by the blob pressure profile itself and study the role of these instabilities in fragmenting the blobs as they transport across the far SOL.

There are two sources of secondary instability in blobs: velocity shear drive for the Kelvin-Helmholtz $(\mathrm{KH})$ instability 23,24 and curvature drive for the sheath-interchange mode. $25,26,2$ In other recent studies of blob stability, it has been shown 27,28 that the $\mathrm{KH}$ instability dominates for very small blobs while the sheath-interchange mode is the most important instability for blobs with $a \gg \rho_{\mathrm{s}}$, where $a$ is the blob radius or typical blob dimension and $\rho_{\mathrm{S}}$ is the ion gyradius. For the blobs of interest here, the inertial term in the vorticity equation (which contains the $\mathrm{KH}$ drive) is unimportant, so we will neglect inertia in most of the discussion. We also make a number of other approximations appropriate to the far SOL, such as neglecting temperature gradients and diamagnetic drifts. A future paper will consider the effects on blob stability of temperature gradients and blob rotation due to the Bohm potential, which may be important in the near SOL.

This paper generalizes our previous model ${ }^{18}$ to include the effect of a background density and of dissipation on the blob equilibrium and stability. For the case of blobs superimposed on a background density, we will show that both the size and the relative height of the blob above background affect its velocity. For a fixed blob size, increasing the blob height relative to the background density produces faster radial propagation; in the limit where the background density vanishes, the maximum velocity [given in Eq. (1)] is obtained. This result agrees with recent simulations by Galkin. ${ }^{29}$ The background density also plays an important role in modifying the blob shape and stability. These trends may be important in understanding the experimental data. The role of dissipation in suppressing the smallest wavelength perturbations is important in understanding how 
coherent, propagating objects can form out of a turbulent background. The role of dissipation in controlling the growth of nonlinear structures was also studied in the context of the Rayleigh-Taylor instability in Ref. 30. In the present paper, the effect of diffusion $\mathrm{D}$ and viscosity $v$ on the secondary instabilities of blobs is analyzed and a simple scaling (valid in the absence of inertia) is described. Finally, the 2D simulations illustrate several features reminiscent of the experimental data, which could not be inferred from simpler models. The 2D model also provides a tool for analyzing the gas puff imaging (GPI) data, which will be discussed in a future paper.

The plan of this paper is the following. In Sec. II we describe the approximations and equations in the model. In Sec. III, the 1D stability analysis is discussed to illustrate the physics of blob stability. In Sec. IV the results of the 2D simulations are presented. Finally, a summary of the main stability results and a discussion of their implications for blob transport is given in Sec. V.

\section{The Model}

\section{A. Basic Equations}

We employ a simple set of dimensionless equations that allows a solution for propagating density blobs 17,18 and their stability. Assuming constant temperature and transforming to a reference frame moving with constant velocity $\mathbf{u}$, we obtain the following vorticity and continuity equations:

$$
\begin{gathered}
\frac{\mathrm{d}}{\mathrm{dt}} \nabla_{\perp}^{2} \varphi+v \nabla_{\perp}^{2} \varphi=\alpha \varphi-\frac{\beta}{\mathrm{n}} \nabla_{\mathrm{y}} \mathrm{n}, \\
\frac{\mathrm{dn}}{\mathrm{dt}}=\mathrm{D} \nabla_{\perp}^{2} \mathrm{n}-\alpha \mathrm{n}, \\
\frac{\mathrm{d}}{\mathrm{dt}}=\frac{\partial}{\partial \mathrm{t}}+\left(\nabla_{\mathrm{x}} \varphi-\mathrm{u}_{\mathrm{y}}\right) \nabla_{\mathrm{y}}-\left(\nabla_{\mathrm{y}} \varphi+\mathrm{u}_{\mathrm{x}}\right) \nabla_{\mathrm{x}} .
\end{gathered}
$$

Here, the potential $\varphi=\Phi-\Phi_{\mathrm{B}}$ is the deviation of the total potential $\Phi$ from the Bohm sheath potential $\left(\Phi=\Phi_{\mathrm{B}} \approx 3 \mathrm{~T}_{\mathrm{e}}\right), \mathbf{v}=\mathbf{b} \times \nabla \varphi$ is the $\mathbf{E} \times \mathbf{B}$ velocity, $\alpha=$ 
$\left(2 \rho_{s} / L_{\|}\right)$measures the net parallel current into the sheaths, $\beta=\left(2 \rho_{s} / R\right)$ is the curvature parameter, $v$ is the viscosity and $\mathrm{D}$ is the particle diffusion coefficient. To make the equations dimensionless, time is normalized to $\Omega_{\mathrm{i}}{ }^{-1}$, length scales to $\rho_{\mathrm{s}}$, and other quantities to reference values (e.g. separatrix values $\mathrm{n}_{\mathrm{s}}$ and $\mathrm{T}_{\mathrm{es}}$ ) so that $\Omega_{\mathrm{i}} \mathrm{dt} \rightarrow \mathrm{dt}$, $\rho_{\mathrm{s}} \nabla \rightarrow \nabla, \mathrm{e} \Phi / \mathrm{T}_{\mathrm{es}} \rightarrow \Phi, \mathrm{v} / \mathrm{c}_{\mathrm{s}} \rightarrow \mathrm{v}, \mathrm{n} / \mathrm{n}_{\mathrm{s}} \rightarrow \mathrm{n}, \mathrm{T}_{\mathrm{e}} / \mathrm{T}_{\mathrm{es}} \rightarrow \mathrm{T}, \mathrm{J} /\left(\mathrm{n}_{\mathrm{s}} \mathrm{ec}_{\mathrm{s}}\right) \rightarrow \mathrm{J}, \mathrm{v} / \Omega_{\mathrm{i}} \rightarrow \mathrm{v}$, $\mathrm{D} /\left(\rho_{\mathrm{s}}^{2} \Omega_{\mathrm{i}}\right) \rightarrow \mathrm{D}$, etc., where $\Omega_{\mathrm{i}}=\mathrm{eB} / \mathrm{m}_{\mathrm{i}} \mathrm{c}$ is the ion cyclotron frequency and $\rho_{\mathrm{s}}=\mathrm{c}_{\mathrm{s}} / \Omega_{\mathrm{i}}$ is the (constant) gyroradius based on $\mathrm{c}_{\mathrm{s}}=\left(\mathrm{T}_{\mathrm{es}} / \mathrm{m}_{\mathrm{i}}\right)^{1 / 2}$.

We assume that the plasma filaments are localized perpendicular to $\mathbf{B}$ and employ a slab model with orthogonal coordinates $(\mathrm{x}, \mathrm{y}, \mathrm{z})$. The $\mathrm{x}$ coordinate is taken in the direction of the major radius $R$, and the dimensionless curvature is written as $\kappa=-\left(\rho_{s} / R\right)$ $\hat{\mathbf{e}}_{\mathrm{x}}$. The $\mathrm{z}$ coordinate is taken along the direction of $\mathbf{B}$, so that $2 \mathbf{b} \times \kappa \cdot \nabla=-\beta \nabla_{\mathrm{y}}$ and $\mathrm{y}$ is approximately in the poloidal direction at the outer midplane. Here, $y=0$ denotes the location of the outer midplane and $\mathrm{x}>0$ corresponds to the SOL, so that motion in the positive $\mathrm{x}$ direction is outwards towards the wall. .

\section{B. Equilibrium and Perturbation Analysis}

We consider the two-dimensional equilibrium and stability problems for a perturbation with wavevector $\mathbf{k}=\mathrm{k}_{\mathrm{x}} \hat{\mathrm{e}}_{\mathrm{x}}+\mathrm{k}_{\mathrm{y}} \hat{\mathrm{e}}_{\mathrm{y}}$ and use the following ansatz:

$$
\begin{aligned}
& \mathrm{n}=\overline{\mathrm{n}}(\mathrm{x}, \mathrm{y}) \mathrm{e}^{-\alpha \mathrm{t}}+\tilde{\mathrm{n}}(\mathrm{x}, \mathrm{y}) \mathrm{e}^{\mathrm{i}(\mathbf{k} \cdot \mathbf{x}-\omega \mathrm{t})} \\
& \varphi=\bar{\varphi}(\mathrm{x}, \mathrm{y})+\tilde{\varphi}(\mathrm{x}, \mathrm{y}) \mathrm{e}^{\mathrm{i}(\mathbf{k} \cdot \mathbf{x}-\omega t)}
\end{aligned}
$$

where overbars denote equilibrium quantities and tildes denote perturbations. Note that the equilibrium is only valid for $\mathrm{t}<\tau_{\|} \sim 1 / \alpha$ (the blob decay time due to parallel losses), which implies that the stability analysis is only valid for modes with growth rates satisfying $\gamma>\alpha$.

The "isolated blob" equilibrium $(\mathrm{d} / \mathrm{dt}=0)$ solution of Eqs. $(2)-(4)$ valid on time scales $\mathrm{t}<<1 / \alpha$ and having a constant radial velocity $\mathrm{u}_{\mathrm{x}}$ is given by 17,18

$$
\bar{n}(x, y)=n_{b} \eta(x) e^{-y^{2} /\left(2 y_{b}^{2}\right)}
$$




$$
\begin{gathered}
\bar{\varphi}(\mathrm{y})=\frac{\beta}{\alpha} \nabla_{\mathrm{y}} \ln \overline{\mathrm{n}}(\mathrm{y})=-\frac{\mathrm{q} y}{\mathrm{y}_{\mathrm{b}}^{2}}, \\
\mathrm{u}_{\mathrm{x}}=-\nabla_{\mathrm{y}} \bar{\varphi}=\frac{\mathrm{q}}{\mathrm{y}_{\mathrm{b}}^{2}}, \quad \mathrm{u}_{\mathrm{y}}=\nabla_{\mathrm{x}} \bar{\varphi}=0,
\end{gathered}
$$

where $\beta / \alpha=L_{\|} / R \equiv q$ was used in Eq. (7). This equilibrium does not have a poloidal velocity because of our assumption that the temperature is independent of $\mathrm{x}$. This solution also neglects the effects of dissipation and a background density profile, which will be included subsequently. It is a useful starting point because it illustrates the effects of blob charge polarization and $\mathbf{E} \times \mathbf{B}$ propagation in their simplest form and leads to the scaling of radial velocity given in Eq. (1).

This solution is valid for an arbitrary function $\eta(x)$. However, it is useful to specify a Gaussian form for $\eta(\mathrm{x})$, so that the equilibrium density has the symmetric form

$$
\bar{n}(x, y)=n_{b} e^{-x^{2} /\left(2 x_{b}^{2}\right)} e^{-y^{2} /\left(2 y_{b}^{2}\right)}
$$

For arbitrary $\mathrm{x}_{\mathrm{b}}$ and $\mathrm{y}_{\mathrm{b}}$ this solution corresponds to an elliptically-shaped blob, and the special case $\mathrm{x}_{\mathrm{b}}=\mathrm{y}_{\mathrm{b}}=a$ reduces to the cylindrical blob ansatz discussed in Ref. 18, $\bar{n}(r)=n_{b} \exp \left[-r^{2} /\left(2 a^{2}\right)\right]$. The general form in Eq. (9) contains the interesting limits of highly-elongated blobs in the radial $\left(\mathrm{x}_{\mathrm{b}} \rightarrow \infty\right)$ or poloidal $\left(\mathrm{y}_{\mathrm{b}} \rightarrow \infty\right)$ directions. We will refer to these cases as "radially-elongated" and "poloidally-elongated" blobs, respectively.

While this equilibrium is useful for analytic studies, it must be generalized to include the effects of a background density and of dissipation to be suitable for a numerical stability analysis. The curvature drive for the sheath-interchange mode is proportional to $(\beta / \alpha)\left(\nabla_{y} \tilde{n}\right) / \bar{n} \propto \mathrm{ik}_{\mathrm{y}}(\tilde{\mathrm{n}} / \overline{\mathrm{n}})$, which becomes singular for the solution in Eq. (9) as $y / y_{b} \rightarrow \infty$ and $\bar{n} \rightarrow 0$. This allows unphysical unstable modes which are localized in the extreme tail of the blob. To eliminate this singularity, we add a constant background or "floor" density $n_{f}$ to $\bar{n}$ : 


$$
\bar{n}(x, y)=n_{b} e^{-x^{2} /\left(2 x_{b}^{2}\right)} e^{-y^{2} /\left(2 y_{b}^{2}\right)}+n_{f}
$$

Here, $\mathrm{n}_{\mathrm{b}}$ is the amplitude of the blob above the floor density at $\mathrm{x}=0=\mathrm{y}$.

The equilibrium solution of Eq. (2) for $\bar{\varphi}$ is then carried out numerically, including both the effects of $\mathrm{n}_{\mathrm{f}}$ and $\mathrm{v}$. The floor density has several effects on the equilibrium. It reduces $\mathrm{u}_{\mathrm{x}}$ below the value predicted in Eq. (1) by reducing the velocity drive term $(\beta / \alpha) \nabla_{y}^{2}(\ln \bar{n}) \approx q / L^{2}$. The density floor introduces a dependence of the density scale length $\mathrm{L}$ and the radial velocity $\mathrm{u}_{\mathrm{x}}$ on the blob height above background, $\delta \mathrm{n}(\mathrm{x}, \mathrm{y})=\overline{\mathrm{n}}(\mathrm{x}, \mathrm{y})-\mathrm{n}_{\mathrm{f}}$. For the $1 \mathrm{D}$ radially-elongated blob, we have $\delta \mathrm{n}=\delta \mathrm{n}(\mathrm{y})$, which introduces a poloidal shear in the radial velocity, $\mathrm{u}_{\mathrm{x}}=\mathrm{u}_{\mathrm{x}}(\mathrm{y})$. This shear has a stabilizing effect on the sheath-interchange mode. For the poloidally-elongated blob, $\bar{\varphi}=0=u_{x}$, so there is no velocity shear. Finally, in the 2D solution of Eq. (2), the spatial variation $\delta \mathrm{n}(\mathrm{x}, \mathrm{y})$ results in a $2 \mathrm{D}$ vortex flow pattern that modifies the blob shape and propagation velocity, as discussed in Sec. IV.

\section{Stability Equations}

Using the ansatz in Eq. (5) and linearizing Eqs. (2) - (4), we obtain the following stability equations for the 1D numerical calculations:

$$
\begin{gathered}
{\left[\gamma+v-i k_{x} u(y)\right] \nabla_{\perp}^{2} \tilde{\varphi}=\alpha \tilde{\varphi}-\beta \nabla_{y} \chi} \\
{\left[\gamma-i k_{x} u(y)\right] \chi=D\left(\frac{1}{\bar{n}}\right) \nabla_{\perp}^{2}(\bar{n} \chi)-i(\mathbf{b} \times \mathbf{k} \cdot \nabla \ln \bar{n}) \tilde{\varphi}}
\end{gathered}
$$

where $\gamma=-i \omega$ is the growth rate, $\chi=(\tilde{\mathrm{n}} / \overline{\mathrm{n}})$ and $\mathrm{u}(\mathrm{y})=\mathrm{u}_{\mathrm{x}}(\mathrm{y}=0)+\bar{\varphi}^{\prime}(\mathrm{y})$ is the shear of the radial velocity. The LHS of the perturbed vorticity equation (11) contains inertial, viscous and velocity shear terms, while the RHS contains the sheath conductivity $(\alpha)$ and curvature $(\beta)$ terms. The linearized density equation (12) balances the inertial and velocity shear terms on the LHS with the diffusion and perturbed $\mathbf{E} \times \mathbf{B}$ convective terms on the RHS.

Some approximations were used in the derivation of these equations. In Eqs. (11) and (12), the stabilizing velocity shear term is written in the form that enters the $1 \mathrm{D}$ 
radially-elongated blob calculation. For the general $2 \mathrm{D}$ problem, we would write $\mathrm{u}_{\mathrm{x}}=$ $\mathrm{u}_{\mathrm{x}}(\mathrm{x}, \mathrm{y})$, but our 2D analysis in Sec. IV is nonlinear and thus does not use the linearized equations; hence, the 1D form of the velocity shear term is sufficient in Eqs. (11) and (12). In deriving the inertial term in Eq. (11), we also assumed $\nabla_{\perp}^{2} \bar{\varphi} \approx 0$, which is strictly true only if $n_{f}=0$. In the numerical results described in the next section it was checked that the inertial term retained in the vorticity equation $(\propto \gamma)$ was negligible for the large blobs $\left(a>>\rho_{\mathrm{S}}\right)$ of interest here, implying that the higher-order corrections to this inertial term are not needed.

It is useful at this point to review the physical picture of the sheath-interchange instability in terms of Eqs. (11) and (12). If the constant density surfaces are perturbed $(\chi \neq 0)$, the curvature drift causes a charge separation and the charges pile up on the perturbed boundary, giving rise to a perturbed potential $(\tilde{\varphi} \neq 0)$. In the simplest limit (neglecting inertia and viscosity) this potential is obtained by balancing the sheath and curvature terms on the RHS of Eq. (11), $\widetilde{\varphi}=(\beta / \alpha) \nabla_{\mathrm{y}} \chi$. The perturbed potential $\tilde{\varphi}$ generates a perturbed electric field in the direction of $\mathbf{k}$, which causes a perturbed $\mathbf{E} \times \mathbf{B}$ drift in the $\mathbf{k} \times \mathbf{b}$ direction, normal to the perturbed density surfaces [last term on the RHS of Eq. (12)]. Instability occurs if this drift reinforces the original density perturbation,

$$
\gamma \chi=-i(\mathbf{b} \times \mathbf{k} \cdot \nabla \ln \bar{n})(\beta / \alpha) \nabla_{y} \chi .
$$

It is important to emphasize that the curvature drive term $[\beta(\mathbf{b} \times \mathbf{k} \cdot \nabla \ln \overline{\mathbf{n}})$ in the present notation] is applied here to the local blob density profile rather than the global SOL profile. The growth of this "secondary instability" drives the nonlinear evolution of the blobs in the simulations and affects the blob size distribution. 


\section{1D Stability Analysis}

In this section, we solve the stability equations (11) and (12) numerically for the equilibrium blob density profile given in Eq. (10) in the limits of poloidally-elongated ( $\mathrm{y}_{\mathrm{b}}$ $\rightarrow \infty)$ and radially-elongated $\left(x_{b} \rightarrow \infty\right)$ blobs. Although the elongation is assumed infinite in each case to obtain a 1D stability calculation, we expect the results to be qualitatively valid for blobs with finite aspect ratio. The 1D calculations will be useful to understand the physics of the instabilities and to introduce the concepts relevant to the $2 \mathrm{D}$ blob simulations discussed in Sec. IV.

\section{A. Poloidally-elongated blobs}

Taking the limit $\mathrm{y}_{\mathrm{b}} \rightarrow \infty, \mathrm{x}_{\mathrm{b}}$ finite in Eq. (10) gives the density profile $\overline{\mathrm{n}}=\overline{\mathrm{n}}(\mathrm{x})$ for poloidally-elongated blobs. It follows from Eq. (7) that $u_{x}=0=u(y)$ because the charge separation occurs over an infinite distance. (This extreme limit is considered only for the stability calculation, and a finite value of $y_{b}$ is assumed when estimating the transport time scale in later sections.) Letting $\nabla \rightarrow \nabla_{\mathrm{x}} \hat{\mathbf{e}}_{\mathrm{x}}+\mathrm{i} \mathrm{k}_{\mathrm{y}} \hat{\mathbf{e}}_{\mathrm{y}}$ in Eqs. (11) and (12) one obtains a set of equations for $\tilde{\varphi}(\mathrm{x})$ and $\chi(\mathrm{x})$ which are solved by a matrix eigenvalue technique after transforming to Fourier space $\left(\nabla_{\mathrm{X}} \rightarrow \mathrm{i} \mathrm{k}_{\mathrm{X}}\right)$.

Before discussing the numerical solution, we first examine the dispersion relation obtained by making the "local approximation" in the linearized equations, valid when $\mathrm{k}_{\mathrm{y}} \mathrm{x}_{\mathrm{b}} \gg 1$. Setting $\mathrm{k}_{\mathrm{x}} \rightarrow 0$ and evaluating $\mathrm{d}(\mathrm{x}) \equiv \mathrm{d} \ln \overline{\mathrm{n}} / \mathrm{dx}$ at the location of the maximum density gradient on the bad curvature side $\left(\mathrm{d}_{\max } \rightarrow-1 / \mathrm{L}\right)$, we obtain

$$
\gamma=-\mathrm{k}_{\mathrm{y}}^{2} \mathrm{D}+\frac{\mathrm{k}_{\mathrm{y}}^{2}(\beta / \mathrm{L})}{\alpha+\mathrm{vk}_{\mathrm{y}}^{2}}=-\mathrm{k}_{\mathrm{y}}^{2} \mathrm{D}+\frac{\mathrm{k}_{\mathrm{y}}^{2}(\beta / \alpha \mathrm{L})}{1+\mathrm{k}_{\mathrm{y}}^{2} \mathrm{a}_{\mathrm{s}}^{2}}
$$

where $a_{s}=(v / \alpha)^{1 / 2}$ is the viscous smoothing radius. In deriving Eq. (14), we have neglected the factor of $\gamma$ from the inertial term in the vorticity equation, because it makes a negligible contribution near marginal stability.

For $\mathrm{D}=0=v$, Eq. (14) gives the local sheath-interchange growth rate $\left(\gamma=\mathrm{k}_{\mathrm{y}}^{2} \beta / \alpha \mathrm{L}\right) .{ }^{25,26}$ The viscosity can reduce the growth rate (when $\mathrm{k}_{\mathrm{y}} \mathrm{a}_{\mathrm{s}}>1$ ) but only 
diffusion can completely stabilize the mode. For $v=0$, the marginal stability boundary is $D=\beta /(\alpha L) \equiv D_{0}$. The dependence of the stability boundary on $D$ and $v$ will be discussed further in Sec. III C. The constant floor density $\mathrm{n}_{\mathrm{f}}$ in Eq. (10) is also stabilizing through its effect on the function $d(x)$ and the local scale length $L=-1 / d_{\max }$. If we set $n_{f}=0$ in Eq. (10), $\mathrm{d}(\mathrm{x}) \propto \mathrm{x}$ increases without bound and the local approximation is invalid; however, $\mathrm{d}(\mathrm{x})$ has a maximum and the value of $\mathrm{L}$ is well-defined when $\mathrm{n}_{\mathrm{f}} \neq 0$. Increasing the value of $n_{f}$ has the effect of increasing $L$ and reducing the instability drive in Eq. (14). These stabilizing effects of $\mathrm{D}, v$ and $\mathrm{n}_{\mathrm{f}}$ are verified by the numerical solutions.

The analytic theory gives the following predictions for the $\mathrm{k}_{\mathrm{y}}$ at marginal stability $\left(\mathrm{k}_{\mathrm{y}}=\mathrm{k}_{0}\right)$ and maximum growth $\left(\mathrm{k}_{\mathrm{y}}=\mathrm{k}_{\max }\right)$ and for the maximum growth rate $\gamma_{\max }$

$$
\begin{gathered}
\mathrm{k}_{0} \mathrm{a}_{\mathrm{s}}=(\Delta-1)^{1 / 2}, \\
\mathrm{k}_{\text {max }} \mathrm{a}_{\mathrm{s}}=\left(\Delta^{1 / 2}-1\right)^{1 / 2}, \\
\gamma_{\max }=\frac{\mathrm{D}}{\mathrm{a}_{\mathrm{s}}^{2}}\left(\Delta^{1 / 2}-1\right)^{2}=\mathrm{Da}_{\mathrm{s}}^{2} \mathrm{k}_{\text {max }}^{4},
\end{gathered}
$$

where $\Delta=\mathrm{D}_{0} / \mathrm{D}=\beta /(\alpha \mathrm{L} \mathrm{D})$. In the limit of weak diffusion $(\Delta>>1)$, we find that $\mathrm{k}_{\mathrm{y}} \mathrm{a}_{\mathrm{s}}>>1$ is satisfied; the condition for the local approximation $\left(\mathrm{k}_{\mathrm{y}} \mathrm{x}_{\mathrm{b}}>>1\right)$ is also satisfied if the viscosity is small enough or the blobs big enough to satisfy the condition $a_{\mathrm{s}}<\mathrm{x}_{\mathrm{b}}$.

For $\mathrm{k}_{\mathrm{y}} \mathrm{x}_{\mathrm{b}} \sim 1$, the non-local mode structure on the scale of the blob must be taken into account by carrying out the numerical solution. Figure 1 shows the non-local eigenfunction $\widetilde{\varphi}(\mathrm{x})$ of the fastest growing mode superimposed on the blob density profile $\overline{\mathrm{n}}(\mathrm{x})$ for typical parameters. The eigenfunction peaks at the maximum density gradient on the bad curvature ( $x>0$ ) side of the blob, and the width of the eigenfunction is comparable to the blob size.

The maximum growth rates $\gamma_{\max }$ for the local and non-local solutions are compared in Fig. 2 (solid curves), where $\gamma_{\max }$ is plotted vs blob size $\mathrm{x}_{\mathrm{b}}$ for fixed values of $\mathrm{D}$ and $v$. The growth rate drops as the blob becomes larger because the pressure gradient 
driving the instability scales like $\beta / \mathrm{x}_{\mathrm{b}}$. The local and non-local solutions show reasonable agreement since $\mathrm{k}_{\mathrm{y}} \mathrm{x}_{\mathrm{b}}>1$ over the range of $\mathrm{x}_{\mathrm{b}}$ shown, and the two solutions converge as $\mathrm{x}_{\mathrm{b}} \rightarrow \infty$ because $\mathrm{k}_{\mathrm{y}} \mathrm{x}_{\mathrm{b}}$ for the most unstable mode increases with $\mathrm{x}_{\mathrm{b}}$. Specifically, the wavenumber ranges from $\mathrm{k}_{\mathrm{y}} \mathrm{x}_{\mathrm{b}}=2.5$ at $\mathrm{x}_{\mathrm{b}}=10$ to $\mathrm{k}_{\mathrm{y}} \mathrm{x}_{\mathrm{b}}=5$ at $\mathrm{x}_{\mathrm{b}}=50$.

The dashed curves $\gamma_{c}\left(x_{b}\right)$ and $\gamma_{c}\left(x_{b}\right) / 10$ in Fig. 2 show the relevant convective time scales and will be discussed in Sec. III C. The competition between the stability and transport time scales illustrated in Fig. 2 is one of the main points of this paper.

\section{B. Radially-elongated blobs}

Taking the other 1D limit ( $\mathrm{y}_{\mathrm{b}}$ finite, $\mathrm{x}_{\mathrm{b}} \rightarrow \infty$ ) in Eq. (10) gives the density profile $\overline{\mathrm{n}}=\overline{\mathrm{n}}(\mathrm{y})$ for radially-elongated blobs, i.e. streamers. As discussed in Sec. II B, a numerical solution is required for $\bar{\varphi}(\mathrm{y})$ to retain the effects of the density floor $\mathrm{n}_{\mathrm{f}}$ and the viscosity $v$ in the vorticity equation. The density floor also sets the maximum of the logarithmic density gradient, $\mathrm{d}(\mathrm{y})$.

The resulting equilibrium solution is illustrated in Fig. 3. Near $y=0$, the numerical solution resembles the analytic solution in Eqs. (7) and (8), viz. $\bar{\varphi}(\mathrm{y})$ linear in $\mathrm{y}$ and $\mathrm{u}_{\mathrm{x}}(\mathrm{y})$ constant, but these functions begin to deviate from their analytic values near the maximum of the density gradient $d(y)$. The functions $d(y)$ and $\bar{\varphi}(y)$ go smoothly to zero as $|\mathrm{y}| \rightarrow \infty$ when $\mathrm{n}_{\mathrm{f}} \neq 0$. The calculation of the growth rate is unaffected by the periodicity boundary condition as long as the domain is much wider than the region of non-zero instability drive $d(y)$. Finally, we see that the density floor and viscosity effects produce a strongly sheared radial velocity $u_{x}(y)$ near the maximum of $d(y)$. This effect enters through the third term on the LHS of Eq. (11) and is stabilizing for the sheathinterchange modes.

Another stabilizing factor is that the curvature is neutral in the radially-elongated blob geometry so there is no local instability analogous to Eq. (14). This is easily seen by letting $\nabla \rightarrow \nabla_{\mathrm{y}} \hat{\mathbf{e}}_{\mathrm{y}}+\mathrm{ik}_{\mathrm{x}} \hat{\mathbf{e}}_{\mathrm{x}}$ in Eqs. (11)-(13) and noting that the RHS of Eq. (13) vanishes because $\mathrm{k}_{\mathrm{y}}=0$. However, the numerical solution shows that there is a weak non-local instability. The physical origin of this instability is illustrated in Fig. 4, where plots of the density weighting $\mathrm{d}(\mathrm{y})$ of the instability drive and the real part of the 
eigenfunction $\widetilde{\varphi}(\mathrm{y})$ are shown. We see that the eigenfunction matches the shape and parity of $\mathrm{d}(\mathrm{y})$ and has enough oscillations to acquire a definite $\mathrm{k}_{\mathrm{y}}$, thereby restoring an instability drive. This instability is non-local because the eigenfunction width is comparable to the blob size; for the case shown $\mathrm{k}_{\mathrm{x}} \mathrm{y}_{\mathrm{b}}=(0.05)(20)=1$. The mode has a complex eigenvalue $\gamma$ with a small real part.

The combination of reduced instability drive and shear flow stabilization leads to weak growth rates (real part of $\gamma$ ) for the radially-elongated blobs, as shown in Fig. 5. Here, we plot the maximum growth rate vs blob size, $\gamma_{\max }\left(y_{b}\right)$. Comparing Figs. 2 and 5, one sees that the growth rate is much weaker in the latter case, both in absolute terms and relative to the transport scales. This leads to the important conclusions that: (1) the blob shape in the 2D simulations is likely to be more elongated in $\mathrm{x}$ than in $\mathrm{y}$; and, (2) the characteristic blob size setting the transport is going to be determined by instabilities with $\mathrm{k}_{\mathrm{y}} \neq 0$.

\section{Implications for 2D stability and transport}

Our analysis of the 1D limiting cases of the linearized stability equations (11) and (12) demonstrated the existence of a "secondary" curvature-driven instability of the blobs due to their internal pressure gradients. The growth rate of this mode depends on the stabilizing effects of sheath conductivity, viscosity, diffusion, and the background density. It was shown that the background density reduces the density gradient weighting of the curvature drive and causes stabilizing sheared flows. The properties of this secondary instability are closely related to the nonlinear behavior of the blob evolution seen in 2D simulation codes, as discussed in Sec. IV.

The 1D growth rate calculations are also useful for comparing the instability and transport time scales for the blobs. The competition between these time scales helps to set the range of blob sizes responsible for the transport. To define the convection time scale, we have to specify the poloidal blob size $\mathrm{y}_{\mathrm{b}}$, which determines the convective velocity $\mathrm{u}_{\mathrm{x}}$ in Eq. (1). At this point the 1D approximation fails us, and we have to take into account the two-dimensional nature of the physical blobs. For simplicity of presentation, we assume isotropy in the following discussion of blob transport time scales. Based on the 
linear stability analysis, we expect the elongation to be of order unity, as discussed subsequently, and this does not substantially affect the present discussion.

Defining a blob radius $a \equiv \mathrm{y}_{\mathrm{b}}=\mathrm{x}_{\mathrm{b}}$, we consider the following characteristic equilibrium and transport times: (i) the time to diffuse one blob radius, $\tau_{\mathrm{d}}=a^{2} / \mathrm{D}$, (ii) the time to convect one blob radius, $\tau_{\mathrm{c}}=a / \mathrm{u}_{\mathrm{x}}=a^{3} / \mathrm{q}$ [by Eq. (8)], and (iii) the time $\tau_{\mathrm{w}}=\Delta \mathrm{x} / \mathrm{u}_{\mathrm{x}}$ to convect to the wall. For purposes of scaling, it is simpler to replace $\tau_{\mathrm{w}}$ by the time to convect a certain number of blob radii; here, for definiteness we assume that the separatrix-wall distance is approximately 10 blob radii (for a typical blob) and replace the convection time to the wall by $10 \tau_{\mathrm{c}}$. We also define the corresponding transport rates $\left(\gamma_{\mathrm{d}}\right.$ $\left.=1 / \tau_{\mathrm{d}}, \gamma_{\mathrm{c}}=1 / \tau_{\mathrm{c}}, \gamma_{\mathrm{w}}=1 / \tau_{\mathrm{w}} \rightarrow \gamma_{\mathrm{c}} / 10\right)$ and note that they scale with blob radius as follows: $\gamma_{\mathrm{d}} \propto 1 / a^{2}$ and $\gamma_{\mathrm{c}} \propto 1 / a^{3}$.

The transport rates and the growth rate $\gamma$ of the most unstable mode are plotted vs blob size for the poloidally- and radially-elongated blobs in Figs. 2 and 5, respectively. It is sufficient to discuss the most unstable situation, viz. the poloidally-elongated blob analysis in Fig. 2, where we now let $\mathrm{x}_{\mathrm{b}} \rightarrow a$. The requirement that the equilibrium be well-defined on the instability time scale requires $\gamma>>\gamma_{d}$. This inequality is satisfied for the range of blob sizes shown, so the curve $\gamma_{\mathrm{d}}(a)$ is omitted from the figure. There are two curve crossings marked by dots in Fig. $2\left(\gamma=\gamma_{c} / 10\right.$ and $\gamma=\gamma_{c}$, corresponding to blob radii $a_{1}$ and $a_{2}$, respectively, which are marked by arrows). For the parameters used in Fig. 2, we find that $a_{1}=10$ and $a_{2}=27$ (in units of gyroradii). These crossing points will be shown to have implications for blob transport.

In discussing the significance of the crossing points, it is useful to introduce the term "essential stability." We say that a blob is "essentially stable" if the fastest growing mode cannot exponentiate in the time it takes the blob to transport a significant distance. To be specific, we choose this distance to be 10 blob radii, so that the condition for essential stability is $\gamma<\gamma_{c} / 10$. By Fig. 2, this criterion is satisfied for $a<a_{1}$. Small blobs are essentially stable to the sheath-interchange instability, whereas large blobs are essentially unstable and break up into smaller ones before moving a significant distance. This process of fragmentation serves to decrease the average radius of the ensemble of 
blobs and to increase its average radial velocity. For blobs with $a>a_{2}$ the instability growth rate is so fast $\left(\gamma>\gamma_{c}\right)$ that the blob breaks up before moving a single blob radius. Thus, the experimentally-measured radial autocorrelation time should be roughly given by $\tau=a_{2} / \mathrm{u}_{\mathrm{x}}\left(a_{2}\right)$ if it is due to blobs transporting between the probes. Blobs in the range $a_{1}<a<a_{2}$ will contribute to the transport while undergoing a cascade to smaller blob sizes. Blobs with $a<a_{1}$ make even greater contributions to the transport without undergoing fragmentation. Determining the size distribution for an ensemble of fragmenting blobs is beyond the scope of this paper, but we expect that it would be primarily populated by blobs in the essentially stable range $a<a_{1}$. Taking $a_{1}$ as an upper bound on the size of the blobs that carry flux to the far SOL and using Eq. (1) to estimate $\mathrm{u}_{\mathrm{x}}$, we find that the corresponding lower limit on the blob radial velocity is given by $\mathrm{u}_{\mathrm{x}}$ > $\mathrm{u}_{\mathrm{x}}\left(a_{1}\right)=(\beta / \alpha) / a_{1}^{2}$.

The assumption of isotropy used above is reasonably consistent with the expected nonlinear evolution of the blobs. The linearized stability analysis suggests that the essentially-stable blobs should be slightly elongated in the radial direction (in the absence of poloidal flows). This result follows if we assume that the nonlinear fragmentation of a blob produces objects whose size is determined by the wavelength of the fastest growing linear mode. For the case of poloidally-elongated blobs, we define the normalized wavevector $\mathrm{K}_{\mathrm{y}}\left(\mathrm{x}_{\mathrm{b}}\right) \equiv \mathrm{k}_{\mathrm{y}} \mathrm{x}_{\mathrm{b}}$ of the fastest growing mode obtained from the linear stability analysis in Fig. 2; we find that $\mathrm{K}_{\mathrm{y}}$ is an increasing function of blob size. For the present estimate, consider the largest essentially-stable blob $\left(\mathrm{x}_{\mathrm{b}}=10\right)$, which has $\mathrm{K}_{\mathrm{y}}=2.5$. A reasonable assumption is that the half-width of the daughter blob corresponds to a quarter wavelength, i.e. $\mathrm{y}_{\mathrm{b}}=\lambda / 4$ where $\lambda=2 \pi / \mathrm{k}_{\mathrm{y}}$. Combining these two relations gives $\mathrm{x}_{\mathrm{b}} / \mathrm{y}_{\mathrm{b}}=$ $2 \mathrm{~K}_{\mathrm{y}} / \pi=5 / \pi \approx 1.6$. For radially-elongated blobs, the linear stability analysis of Fig. 5 gives the value of $k_{x} y_{b} \equiv K_{x}\left(y_{b}\right)$ for the fastest growing mode. The value of $K_{x}$ increases with blob size and $K_{x}>1.0$ for $y_{b}>25$. The case shown in Figs. 3 and $4\left(y_{b}=30\right)$ is close to the largest essentially-stable radial blob and has $K_{x}=1.5$. Assuming $x_{b}=\lambda / 4$ where $\lambda=2 \pi / \mathrm{k}_{\mathrm{x}}$ leads to the result that $\mathrm{x}_{\mathrm{b}} / \mathrm{y}_{\mathrm{b}}=\pi /\left(2 \mathrm{~K}_{\mathrm{x}}\right)=\pi / 3.0 \approx 1.0$, which implies isotropy. The instabilities of the radially-elongated blobs/streamers have longer 
wavelengths than those of the poloidally-elongated case; moreover, the growth rates are so weak that the effects of these modes may not actually be observed in the simulations. Thus, the two 1D calculations taken together suggest that the blobs will have a radial elongation $\mathrm{x}_{\mathrm{b}} / \mathrm{y}_{\mathrm{b}}$ in the range 1-1.6, similar to what is observed in the $2 \mathrm{D}$ simulations discussed in the next section. However, sheared poloidal flows (neglected in our 1D and 2D models) may also affect the final blob shape by stretching the blobs in the poloidal direction. In the presence of these flows, the blob shape would be determined by a competition between the sheared poloidal flow and instability time scales.

In the calculations discussed so far, the dissipation coefficients D and $v$ were held fixed as the blob size was varied. We have also investigated the dependence of the stability and transport on these parameters. In the absence of inertia, it can be shown from either the nonlinear Eqs. (2) and (3) or the linearized Eqs. (16) and (17), that the blob radius $a$ can be scaled out of the stability problem by the transformations

$$
\mathrm{D} \rightarrow a \mathrm{D} \equiv \hat{\mathrm{D}}, \quad \mathrm{a}_{\mathrm{s}} \rightarrow \mathrm{a}_{\mathrm{s}} / a \equiv \hat{\mathrm{a}}_{\mathrm{s}}, \quad \gamma \rightarrow \gamma a^{3} \equiv \hat{\gamma}, \varphi \rightarrow a \varphi \equiv \hat{\varphi}, \mathrm{n} \rightarrow \mathrm{n} \equiv \hat{\mathrm{n}}
$$

where $\mathrm{n}$ and $\varphi$ denote either the equilibrium or perturbed potentials. Also note that $\hat{\gamma} \propto \gamma / \gamma_{c}$. It is interesting that the normalization of the growth rate to the convective time emerges naturally from this scaling. The crossing points discussed previously correspond to $\hat{\gamma}=q / 10$ and q. In Fig. 6 we plot the equilibrium, stability and transport boundaries in the $\left(\hat{a}_{\mathrm{s}}, \hat{\mathrm{D}}\right)$ plane for the poloidally-elongated blob with $\mathrm{q}=3$. The growth rates are calculated using the local dispersion relation in Eq. (14). The solid curves give the growth rate contours $\hat{\gamma}=0, \mathrm{q} / 10$ and $\mathrm{q}$, corresponding to $\gamma / \gamma_{\mathrm{c}}=0,0.1$ and 1 , respectively. The curve farthest on the right is the marginal stability boundary $(\hat{\gamma}=0)$. The region of small $\hat{\mathrm{a}}_{\mathrm{s}}$ and $\hat{\mathrm{D}}$ below this curve is unstable and the growth rate increases towards the origin; the region above the marginal stability curve at large $\hat{a}_{s}$ and $\hat{D}$ is stable. Moving leftwards from the $\hat{\gamma}=0$ curve, the other two solid curves correspond to $\hat{\gamma}=q / 10$ and $q$, respectively, which give the trajectories of the crossing points $a_{1}$ and $a_{2}$ in the $\left(\hat{\mathrm{a}}_{\mathrm{S}}, \hat{\mathrm{D}}\right)$ plane. Using the 10 blob-radius criterion, the region of essential stability is the region above the curve $\hat{\gamma}=q / 10$. Fast blob fragmentation (before moving one blob radius) 
occurs below the curve $\hat{\gamma}=\mathrm{q}$. Also shown is the upper limit on $\hat{\mathrm{D}}$ imposed by the requirement that the blob convect faster than it spreads due to diffusion $\left(\gamma_{d}<\gamma_{c}\right)$. Thus, Fig. 6 generalizes the concepts illustrated in Fig. 2 and is useful for guiding the parameter choices for the 2D simulations in the next section.

\section{2D Simulation Results}

We have carried out 2D simulations of blob behavior in which the nonlinear Eqs. (2) - (4) are integrated in time. This approach is valid even when the blob is not in equilibrium (e.g. in the limit of large diffusion) and it allows investigation of the nonlinear evolution of the blobs. The $2 \mathrm{D}$ simulations confirm the main points from the 1D stability analyses and illustrate qualitative features that may relate to the experimental observations.

To simplify the simulation algorithm, the inertial term is neglected in Eq. (2); this approximation is valid for sufficiently large blobs satisfying the condition $(\mathrm{k} a)^{4}<<\alpha a^{5}$, where $\mathrm{k}$ is the wavenumber of the fastest growing mode and $a$ is the blob radius (assuming a cylindrical blob). The ratio of the parameters $\alpha$ and $\beta$ is fixed for all the results shown here $(q=\beta / \alpha=1)$ and the viscosity is specified in terms of the smoothing radius $a_{s}$. Finally, we neglect parallel particle losses, valid for timescales $t<<1 / \alpha$, to focus attention on the physics of instability and dissipation.

In Fig. 7, the growth of a robust interchange instability with $\mathrm{k}_{\mathrm{y}} \neq 0$ is illustrated for a blob propagating through a very small background density $\left(\mathrm{n}_{\mathrm{f}}=0.01\right)$ in the

presence of weak dissipation $\left(\mathrm{D}=0.01, \mathrm{a}_{\mathrm{S}}=10^{-4}\right.$ ). The blob was initialized at $\mathrm{t}=0$ using the functional form in Eq. (10) with $\mathrm{n}_{\mathrm{b}}=1$ and $a=\mathrm{x}_{\mathrm{b}}=\mathrm{y}_{\mathrm{b}}=10$. The mode has grown to a large enough amplitude to break up the blob after a few convection times $(\mathrm{t}=$ $1.75 \tau_{\mathrm{c}}$ ), where $\tau_{\mathrm{c}}=a / \mathrm{u}_{\mathrm{x}}$ with $\mathrm{u}_{\mathrm{x}}$ given by Eq. (1). In this case, the nonlinear instability grows fast enough to affect the transport.

Consistent with the 1D stability analysis, essentially stable blob propagation is possible in the $2 \mathrm{D}$ simulations by using a combination of diffusion, viscosity, and a background density to reduce the growth rate. Large diffusion causes the blob to spread 
as it propagates, which is not what is observed in the experiments. Thus, we use a small value of the diffusion coefficient in the examples shown here and rely on a combination of viscosity and background density to stabilize the blobs.

The stabilizing effect of the background density is illustrated in Fig. 8 for the parameters $\mathrm{D}=0.01$ and $\mathrm{a}_{\mathrm{S}}=0$ (no viscosity). Again the blob was initialized at $\mathrm{t}=0$ using Eq. (10) with $\mathrm{n}_{\mathrm{b}}=1$ and $a=\mathrm{x}_{\mathrm{b}}=\mathrm{y}_{\mathrm{b}}=10$. In this figure, we compare the evolution of a blob for two values of the background density: (a) $n_{f}=0.1$ and (b) $n_{f}=0.5$. Case (a) is clearly in the nonlinear phase of the instability at $t=3.8 \tau_{c}$, whereas case (b) does not yet exhibit instability at the same point in time. Figure 8 shows that increasing the background density for fixed blob height $\mathrm{n}_{\mathrm{b}}$ slows down the blob propagation and increases its stability.

The influence of the background density on the blob shape and velocity is illustrated for a different series of runs in Figs. 9 and 10. This simulation has non-zero viscosity $\left(a_{S}=7\right)$ but small diffusion $(D=0.005)$; the other parameters are given in the figure caption. Figure 9 shows a comparison of the blob evolution after many convection times $\left(\mathrm{t}=12.5 \tau_{\mathrm{c}}\right)$ for three values of the background density. In the case with the smallest background density $\left(\mathrm{n}_{\mathrm{f}}=0.01\right)$, the blob is unstable and has bifurcated in the course of the simulation; at the time shown, the two daughter blobs are poloidally separated and have become radially elongated. The two cases with higher background density $\left(\mathrm{n}_{\mathrm{f}}=0.1\right.$ and 0.5$)$ are still stable after more than $10 \tau_{\mathrm{c}}$. Also note that the radial velocity and the distortion in shape are largest for the case with the smallest background density. The radial elongation of the blobs is due to the development of a sheared flow pattern, which steepens the leading edge and produces a wake at the trailing edge of the blob (see Fig. 9). Reducing the density floor in the simulation speeds up the blob [toward the theoretical value in Eq. (1)], increases the distortion in its shape, and decreases its stability, leading to bifurcation. (However, it should be recalled from our earlier discussion in Sec. II B that removing the density floor entirely would eliminate the sheared flow which produces the distortion in blob shape. The limit $\mathrm{n}_{\mathrm{f}} \rightarrow 0$ is singular, as discussed previously, because in that case $\varphi \propto \nabla_{\mathrm{y}} \mathrm{n} / \mathrm{n}$ is unbounded for large $|\mathrm{y}|$.) Finally, 
we note that the development of a steep leading edge followed by a long trailing wake is a universal feature in the experimental probe data. $7,12,31$

The effect of $n_{f}$ on the blob velocity $u_{x}$ for these cases is shown in Fig. 10. The blob displacement $\mathrm{x}(\mathrm{t})$ is plotted as a function of time for the analytic result [Eq. (1)] in the absence of diffusion, viscosity or background density (dashed curve in Fig. 10), and for the three cases of Fig. 9 with varying background densities (solid curves in Fig. 10). Compared with the analytic value, the velocity $u_{x}$ is reduced in the simulations because of the effects of both the density floor and the viscosity. The viscosity is held fixed in the three simulations shown here, so the change in slope of the curves in Fig. 10 is due entirely to the background density. The time history in the unstable case $\left(n_{f}=0.01\right)$ shows a very interesting cusp-like behavior. We see that the parent blob slows down before bifurcating and the smaller daughter blob accelerates after the bifurcation. The details of this bifurcation will be shown in more detail in Fig. 11. The increased slope in Fig. 10 after bifurcation, which indicates a larger radial velocity, is qualitatively consistent with the theoretical scaling of $\mathrm{u}_{\mathrm{x}}$ with poloidal blob size in Eq. (1).

In Fig. 11, we illustrate the physics of blob bifurcation in more detail. This figure shows four frames in the time history of the case described in Fig. 9(a) and Fig. 10(b). The initial blob in Fig. 11(a) is circular. In Fig. 11(b) the growth of an instability with $\mathrm{k}_{\mathrm{y}}$ $\neq 0$ is evident. The bean shape of the blob in this frame (with two wings projected forwards) is universally seen in the simulations as the secondary instability develops and is probably associated with the background sheared flows seeding the instability. The nonlinear development of the instability in frames (c) and (d) leads to a complete bifurcation of the initial blob into two radially-elongated blobs. The end result of this simulation is consistent with the discussion in Sec. III, where it was shown that the strongest instability is driven by poloidal modulations of the density $\left(\mathrm{k}_{\mathrm{y}} \neq 0\right)$, suggesting that 2D blobs will be either isotropic in shape or somewhat radially-elongated.

The behavior of the radial velocity $\mathrm{u}_{\mathrm{x}}$ in Fig. 10(b) can be understood from the evolution of the blob shape in Fig. 11. We see that the blob elongates in the poloidal direction [Fig. 11(b)] before it bifurcates and then compresses in the poloidal direction 
after bifurcation as it elongates radially [Fig. 11(c)]. This behavior accounts for the slowdown before and speed-up after bifurcation observed in Fig. 10(b). Also, note that the radial velocity is independent of the radial elongation because the charge polarization driving the outwards drift occurs in the poloidal direction.

Finally, in Fig. 12 we show the interaction of a small and a larger blob traveling at the same poloidal location. Since a small blob travels faster than a large one, the small one catches up to the larger one if it starts out behind it. The result is a merger, as shown in Fig. 12. This run uses substantial diffusion and viscosity so that the large blob is stable without a significant background density. The effect of diffusion on the blobs is evident on the long time scale shown $\left(t=0.62 \tau_{d}\right)$.

\section{Summary and Discussion}

The blob model provides a natural mechanism for SOL particle transport. One of the key features of the model is that the radial velocity depends on the poloidal blob size [Eq. (1)], because the charge separation giving rise to the $\mathbf{E} \times \mathbf{B}$ drift occurs over this distance. In this paper, we have shown that the poloidal blob size distribution is modified by secondary instabilities of the sheath-interchange type, driven by curvature and weighted by the blob pressure profile. The fastest growing interchange instabilities can be "global" on the scale of the blob and cause fragmentation of the blobs into smaller objects which propagate more rapidly than the original ones, thereby increasing the radial particle transport. The evolution of the blob size distribution due to instabilities and its effect on the radial transport is one of the main points of this paper.

A survey of the main dependences of the growth rate was carried out in Sec. III using one-dimensional models (assuming infinite elongation in either the radial or poloidal directions). It was shown that poloidally-elongated blobs were unstable to both

local and non-local modes with $\mathrm{k}_{\mathrm{y}} \neq 0$, whereas radially-elongated blobs were only weakly unstable to non-local modes with $\mathrm{k}_{\mathrm{X}} \neq 0$. For the fastest growing modes in the poloidally-elongated case, $\mathrm{k}_{\mathrm{y}} \mathrm{x}_{\mathrm{b}}$ is order unity, which suggests that the blobs tend toward isotropy as they go unstable. Applying these results to two-dimensional cylindrical blobs 
with radius $a$, the growth rate was compared with typical diffusive and convective time scales in Fig. 2, discussed in Sec. III C. It was shown that the competition between the stability and transport time scales leads to two characteristic blob radii. First, there is a critical blob radius $\left(a=a_{1}\right)$ below which the blob is essentially stable during the time it takes to convect to the wall, whereas larger blobs $\left(a>a_{1}\right)$ break up before reaching the wall. Thus, the requirement of interchange stability puts an upper bound on the blob radii $\left(a<a_{1}\right)$, and a corresponding lower bound on the blob radial velocities $\left[\mathrm{u}_{\mathrm{x}}>(\beta / \alpha) / a_{1}^{2}\right]$, contributing to transport. Very large blobs $\left(a>a_{2}\right)$ go unstable and break up before moving one blob radius, so they will probably not be observed in the experiment. This implies that the experimentally-measured radial autocorrelation time should be roughly given by $\tau=a_{2} / \mathrm{u}_{\mathrm{x}}\left(a_{2}\right)$ if it is due to blobs transporting between the probes. This estimate is in factor of 2 agreement with the measured autocorrelation times.

We mention in passing another application of the poloidally-elongated blob stability analysis. By giving a "preferred" (e.g. most unstable) wavenumber $\mathrm{k}_{\mathrm{y}}$, it motivates the use of projection onto a restricted set of wavenumbers (e.g. $\mathrm{k}_{\mathrm{y}}=0$ and $\mathrm{k}_{\mathrm{y}}=$ $\mathrm{k}_{\max }$ ) as an approximation technique for reducing the simulation problem to a set of 1D radial equations. This technique has been used for studying bursty transport ${ }^{32}$ and for studying electromagnetic corrections to blob theory and its application to the density limit problem. ${ }^{33}$

The results of 2D simulations of blob dynamics were described in Sec. IV. The 2D simulations verify the instability physics described in the linearized 1D models and extend the treatment to the nonlinear regime. The secondary instabilities have the effect of reducing the typical blob size and increasing the transport in the simulations. The analytic scaling of radial velocity with poloidal blob size is recovered in the appropriate limits and extended here to include the effects of dissipation and of a background density. The simulations show a rich variety of blob dynamics, including bifurcations and mergers, which is reminiscent of the images seen in 3D turbulence simulations ${ }^{21}$ and in the GPI sequences. ${ }^{11,13}$ 
An important result of the present work was the investigation of the dependence of the blob shape, velocity and stability on the background plasma density, which is neglected in the analytic density blob model. ${ }^{17,18}$ [See the discussions after Eq. (10) in Sec. II and of Figs. 9 and 10 in Sec. IV]. The background density increases the density scale length L, especially in the low-density tail. This effect reduces the charge polarization, which drives the radial velocity and the linear instability drive, thereby slowing down the blob and helping to suppress its break-up. The background density also modifies the blob shape, creating a steep leading edge and a trailing wake, which causes the blob to elongate in the radial direction. All of these effects are sensitive to the blob amplitude above the background, $\delta \mathrm{n}=\overline{\mathrm{n}}-\mathrm{n}_{\mathrm{f}}$; the analytic blob model is recovered in the vacuum limit $\left(n_{\mathrm{f}} \rightarrow 0\right)$ and the blob transport becomes unimportant in the limit of low amplitude $(\delta \mathrm{n} \rightarrow 0)$.

The effects of the background density may be important in comparing theory with experimental data. In the near SOL, there is a significant background density due to diffusion of particles from the core, but this background density profile $n_{\mathrm{f}}(\mathrm{x})$ is observed to decay more rapidly than the blob density profile $\overline{\mathrm{n}}(\mathrm{x}){ }^{7,12}$ As the blobs move further into the SOL, the present work suggests that the decay of the background density should cause the blobs to speed up, to break up into smaller fragments (which further enhances their transport), and to become less elongated radially.

A number of physical effects were omitted from the present work and should be included in a complete theory. Fine-scaled Kelvin-Helmholtz instabilities driven by velocity shear were not discussed here but have been shown to play a role for small blobs in other recent simulations. ${ }^{27,28}$ Including these modes requires a proper treatment of the inertial term in Eq. (2). The present model also neglects temperature gradients in the blob and the background plasma, and thus omits blob rotation due to the Bohm potential $\left(\Phi \approx 3 \mathrm{~T}_{\mathrm{e}}\right)$ and diamagnetic flow $\mathrm{u}_{\mathrm{y}}(\mathrm{x})$. Including temperature-gradient effects may explain the near-isotropy of blob shapes observed in the GPI data. Extensions of the model to include these effects and comparisons of the model with experimental data will be the subject of future work. 


\section{Acknowledgements}

We thank S. Krasheninnikov and S. Galkin for several useful discussions of blob stability; J. Boedo, S. Zweben, R. Maqueda, and J. Terry for discussions of the experimental data; and G. Yu for providing a preprint of his work. This work was supported by the U.S. Department of Energy (DOE) under DOE Grant No. DE-FG0397ER54392; however, this support does not constitute an endorsement by the DOE of the views expressed herein. 


\section{References}

${ }^{1}$ S. J. Zweben, Phys. Fluids 28, 974 (1985).

${ }^{2}$ M. Endler, H. Niedermeyer, L. Giannone, E. Holzhauer, A. Rudyj, G. Theimer, N. Tsois and ASDEX team, Nucl. Fusion 35, 1307 (1995).

${ }^{3}$ R. A. Moyer, R. D. Lehmer, T. E. Evans, R. W. Conn, and L. Schmitz, Plasma Phys. Contr. Fusion 38, 1273 (1996).

${ }^{4}$ M. Umansky, S. I. Krasheninnikov, B. LaBombard and J. L. Terry, Phys. Plasmas 5, 3373 (1998); B. LaBombard, M. V. Umansky, R. L. Boivin, J. A. Goetz, J. Hughes, B. Lipschultz, D. Mossessian, C. S. Pitcher, J. L. Terry, Alcator Group, Nucl. Fusion 40, 2041 (2000).

${ }^{5}$ G. Y. Antar, P. Devynck, X. Garbet, and S. C. Luckhardt, Phys. Plasmas 8, 1612 (2001).

${ }^{6}$ G. Y. Antar, S. I. Krasheninnikov, P. Devynck, R. P. Doerner, E. M. Hollmann, J. A. Boedo, S. C. Luckhardt and R. W. Conn, Phys. Rev. Letters 87, 065001 (2001).

${ }^{7}$ J. Boedo, D. Rudakov, R. Moyer, S. Krasheninnikov, D. Whyte, G. McKee, G. Tynan, M. Schaffer, P. Stangeby, et al., Phys. Plasmas 8, 4826 (2001).

${ }^{8}$ S. Zweben, D. P. Stotler, J. L. Terry, B. LaBombard, M. Greenwald, et al., Phys. Plasmas 9, 1981 (2002).

${ }^{9}$ D. L. Rudakov, J. A. Boedo, R. A. Moyer, S. Krasheninnikov, A. W. Leonard, et al., Plasma Phys. Contr. Fusion 44, 717 (2002).

${ }^{10}$ J. Neuhauser, D. Coster, H. U. Fahrbach, J. C. Fuchs, G. Haas, et al., Plasma Phys. Contr. Fusion 44, 855 (2002).

${ }^{11}$ J. L. Terry et al., APS 2002 Invited Paper KI2.001, to be published in Phys. Plasmas (2003).

${ }^{12}$ J. A. Boedo et al., APS 2002 Invited Paper KI2.002, to be published in Phys. Plasmas (2003).

${ }^{13}$ S. Zweben, R. Maqueda, D.P. Stotler, A. Keesee, J. Boedo et al., to be published in Nucl. Fusion (2003). 
${ }^{14}$ A. Yu. Pigarov, S. I. Krasheninnikov, T. D. Rognlien, M. J. Schaffer, and W. P. West, Phys. Plasmas 9, 1287 (2002).

${ }^{15}$ S. I. Krasheninnikov, S. A. Galkin, A. Yu. Pigarov, D. A. D’Ippolito, J. R. Myra, D. R. McCarthy, W. M. Nevins, T. D. Rognlien, X. Q. Xu, et al., in Proceedings of the 19th IAEA Fusion Energy Conference, Lyon, France, paper TH/4-1 (2002).

${ }^{16}$ M. J. Greenwald, Plasma Phys. Contr. Fusion 44, R27 (2002).

${ }^{17}$ S. I. Krasheninnikov, Phys. Lett. A, 283, 368 (2001).

${ }^{18}$ D. A. D’Ippolito, J. R. Myra, and S. I. Krasheninnikov, Phys. Plasmas 9, 222 (2002).

${ }^{19}$ R.J. Maqueda, G. A. Wurden, S. J. Zweben, L. Roquemore, S. Kaye, D. P. Stotler, et al., Bull. APS 46, 262 (2001), paper QP1 27.

${ }^{20}$ A. V. Nedospasov, V. G. Petrov, and G. N. Fidel'man, Nucl. Fusion 25, 21 (1985).

${ }^{21}$ X. Xu, W M Nevins, R H Cohen, J R Myra and P B Snyder, New Journal of Physics 4, 53.1-53.15 (2002) (http://www.njp.org/).

${ }^{22}$ J. R. Myra, D. A. D’Ippolito, X. Q. Xu, and R. H. Cohen, Contrib. Plasma Phys. 40, 352 (2000); J. R. Myra, D. A. D’Ippolito, X. Q. Xu, and R. H. Cohen, Phys. Plasmas 7, 4622 (2000), and references therein.

${ }^{23}$ S. Chandrasekhar, Hydrodynamic and Hydromagnetic Stability (Oxford University Press, New York, 1961), Chap. XI.

${ }^{24}$ P. Pritchett, Phys. Fluids 30, 272 (1987).

${ }^{25}$ A. V. Nedospasov, Fiz. Plazmy 15, 1139 (1989) [Sov. J. Plasma Phys. 15, 659 (1989)].

${ }^{26}$ X. Garbet, L. Laurent, J.-P. Roubin, and A. Samain, Nucl. Fusion 31, 967 (1991).

${ }^{27}$ N. Bian, S. Benkadda, J-V. Paulsen, and O. E. Garcia, Phys. Plasmas 10, 671 (2003).

${ }^{28}$ G. Q. Yu, S. A. Galkin, S. I. Krasheninnikov, and A. Yu Pigarov, submitted to Phys. Plasmas (2003).

${ }^{29}$ S. A. Galkin, S. I. Krasheninnikov, D. A. D’Ippolito, J. R. Myra, X. Q. Xu, Bull. APS 47, 176 (2002), paper GP1 149.

${ }^{30}$ A. Das, A. Sen, S. Mahajan, and P. Kaw, Phys. Plasmas 8, 5104 (2001).

${ }^{31}$ G. Y. Antar, G. Counsell, Y. Yu, B. LaBombard, P. Devynck, Phys. Plasmas 10, 419 (2003). 
${ }^{32}$ S. Benkadda, P. Beyer, N. Bian, C. Figarella, O. Garcia, X. Garbet, P. Ghendrih. Y. Sarazin, and P. H. Diamond, Nucl. Fusion 41, 995 (2001).

${ }^{33}$ J. R. Myra, D. A. D'Ippolito, S. I. Krasheninnikov and S. A. Galkin, in Proceedings of the 29th EPS Conference on Plasma Physics and Controlled Fusion, Montreux, Switzerland (2002). 


\section{Figure Captions}

Fig. 1 Plots of the density profile $\overline{\mathrm{n}}(\mathrm{x})$ of the poloidally-elongated blob (solid curve, labeled " $n ")$ and the normalized eigenfunction $\tilde{\varphi}(\mathrm{x})$ of the most unstable mode (dashed curve, labeled "phi") for $\mathrm{k}_{\mathrm{y}}=0.25, \mathrm{x}_{\mathrm{b}}=10, \mathrm{D}=0.001, \mathrm{v}=0.1, \mathrm{q}=3$, and $\mathrm{n}_{\mathrm{f}}=0.01$.

Fig. 2 Plots of the dimensionless instability growth rate and transport rates vs radial blob size $\mathrm{x}_{\mathrm{b}}$ for poloidally-elongated blobs. Here, $\gamma$ and $\mathrm{x}_{\mathrm{b}}$ are in units of $\Omega_{\mathrm{i}}$ and $\rho_{\mathrm{s}}$, respectively. Shown are (a) $\gamma_{\max }\left(\mathrm{x}_{\mathrm{b}}\right)$ for the non-local numerical solution (lower solid curve), (b) $\gamma_{\max }\left(x_{b}\right)$ for the local analytic solution (upper solid curve), (c) $\gamma_{c}\left(x_{b}\right) / 10$ (lower dashed curve) and (d) $\gamma_{c}\left(x_{b}\right)$ (upper dashed curve). The parameters are $\mathrm{D}=0.001, \mathrm{v}=0.1, \mathrm{q}=3$ and $\mathrm{n}_{\mathrm{f}}=0.01$. The dots and arrows indicate the crossings of the stability and transport curves at $\mathrm{x}_{\mathrm{b}}=a_{1}$ and $a_{2}$, as explained in the text.

Fig. 3 Equilibrium profiles for the radially-elongated blob. Shown are the density weighting $d(y)=\bar{n}^{\prime}(y) / \bar{n}$ for the curvature drive (solid curve, labeled "d"), the equilibrium potential $\bar{\varphi}(\mathrm{y})$ (short-dashed curve, labeled "phi"), and the radial velocity $\mathrm{u}_{\mathrm{x}}(\mathrm{y})$ with arbitrary normalization (long-dashed curve, labeled "u") for the parameters $\mathrm{y}_{\mathrm{b}}=30, \mathrm{D}=0.001, \mathrm{v}=0.1, \mathrm{q}=3$, and $\mathrm{n}_{\mathrm{f}}=0.01$.

Fig. 4 Comparison of $\mathrm{d}(\mathrm{y})=\overline{\mathrm{n}}^{\prime}(\mathrm{y}) / \overline{\mathrm{n}}$ with arbitrary normalization (solid line, labeled "d") and the real part of the most unstable eigenfunction $\widetilde{\varphi}(\mathrm{y})$ (dashed line, labeled "phi") for the radially-elongated blob with parameters $\mathrm{k}_{\mathrm{x}}=0.05, \mathrm{y}_{\mathrm{b}}=20$, $\mathrm{D}=0.001, \mathrm{v}=0.1, \mathrm{q}=3$ and $\mathrm{n}_{\mathrm{f}}=0.01$.

Fig. 5 Plots of the dimensionless instability growth rate and transport rates vs poloidal blob size $y_{b}$ for radially-elongated blobs. Here, $\gamma$ and $y_{b}$ are in units of $\Omega_{i}$ and $\rho_{s}$, respectively. Shown are (a) $\gamma_{\max }\left(y_{b}\right)$ for the non-local numerical solution (solid curve), (b) $\gamma_{c}\left(y_{b}\right) / 10$ (lower dashed curve), and (c) $\gamma_{c}\left(y_{b}\right)$ (upper dashed curve). The parameters are $\mathrm{D}=0.001, \mathrm{v}=0.1, \mathrm{q}=3$ and $\mathrm{n}_{\mathrm{f}}=0.01$. The arrow indicates the crossing of the stability and transport curves. 
Fig. 6 Equilibrium, stability and transport boundaries in the $\left(\hat{a}_{s}, \hat{D}\right)$ plane for poloidallyelongated blobs with $\mathrm{q}=3$. The parameters $\hat{\mathrm{a}}_{\mathrm{s}}=\mathrm{a}_{\mathrm{s}} / a$ and $\hat{\mathrm{D}}=a \mathrm{D}$ scale the blob radius $a$ out of the stability problem.

Fig. 7 Strongly unstable density blob in the nonlinear phase. The blob was initialized using Eq. (10) with $\mathrm{x}_{\mathrm{b}}=\mathrm{y}_{\mathrm{b}}=10, \mathrm{n}_{\mathrm{b}}=1$ and $\mathrm{n}_{\mathrm{f}}=0.01$; the other parameters are $\mathrm{D}$ $=0.01, \mathrm{a}_{\mathrm{s}}=10^{-4}$, and $\mathrm{t}=1.75 \tau_{\mathrm{c}}$.

Fig. 8 Blob propagating on a floor density $\mathrm{n}_{\mathrm{f}}$ of (a) 0.1 and (b) 0.5 after the elapsed time $\mathrm{t}=3.8 \tau_{\mathrm{c}}$. The blob was initialized using Eq. (10) with $\mathrm{x}_{\mathrm{b}}=\mathrm{y}_{\mathrm{b}}=10$ and $\mathrm{n}_{\mathrm{b}}=1$; the other parameters are $\mathrm{D}=0.01$ and $\mathrm{a}_{\mathrm{s}}=0$. Case (a) is clearly in the nonlinear phase $(\gamma t>1)$ whereas case $(b)$ is not yet unstable.

Fig. 9 Blob propagating on a floor density $n_{f}$ of (a) 0.01 , (b) 0.1 and (c) 0.5 for the parameters $\mathrm{D}=0.005$ and $\mathrm{a}_{\mathrm{s}}=7$. The blob was initialized using Eq. (10) with $\mathrm{x}_{\mathrm{b}}$ $=\mathrm{y}_{\mathrm{b}}=10$ and $\mathrm{n}_{\mathrm{b}}=1$. The elapsed time is $\mathrm{t}=12.5 \tau_{\mathrm{c}}$.

Fig. 10 Blob displacement $x$ vs $t / \tau_{c}$ for the analytic result in Eq. (1) with $D=a_{s}=n_{f}=0$ (dashed line), and the three cases shown in Fig. 9 (solid curves). The latter cases have a floor density $\mathrm{n}_{\mathrm{f}}$ of $0.01,0.1$, and 0.5 , respectively, with $\mathrm{D}=0.005$ and $\mathrm{a}_{\mathrm{s}}=$ 7. Note that the $\mathrm{n}_{\mathrm{f}}=0.01$ case exhibits slow-down before bifurcation and speedup afterwards. The reduced $u_{x}$ for the solid curves is due to both the effects of non-zero background density and viscosity.

Fig. 11 Blob propagation with finite viscosity showing weak instability and subsequent bifurcation for the case $n_{f}=0.01$ in Fig. 10 at four values of $t / \tau_{c}$ : (a) 0 , (b) 6 , (c) 9 , and (d) 12 .

Fig. 12 Merger of a small and large blob for the parameters $D=0.005, a_{s}=10$ and $n_{f}=$ 0.01. The simulation results are shown at three values of $t / \tau_{\mathrm{c}}$ : (a) 0 , (b) 4.5 , and (c) 9 . 


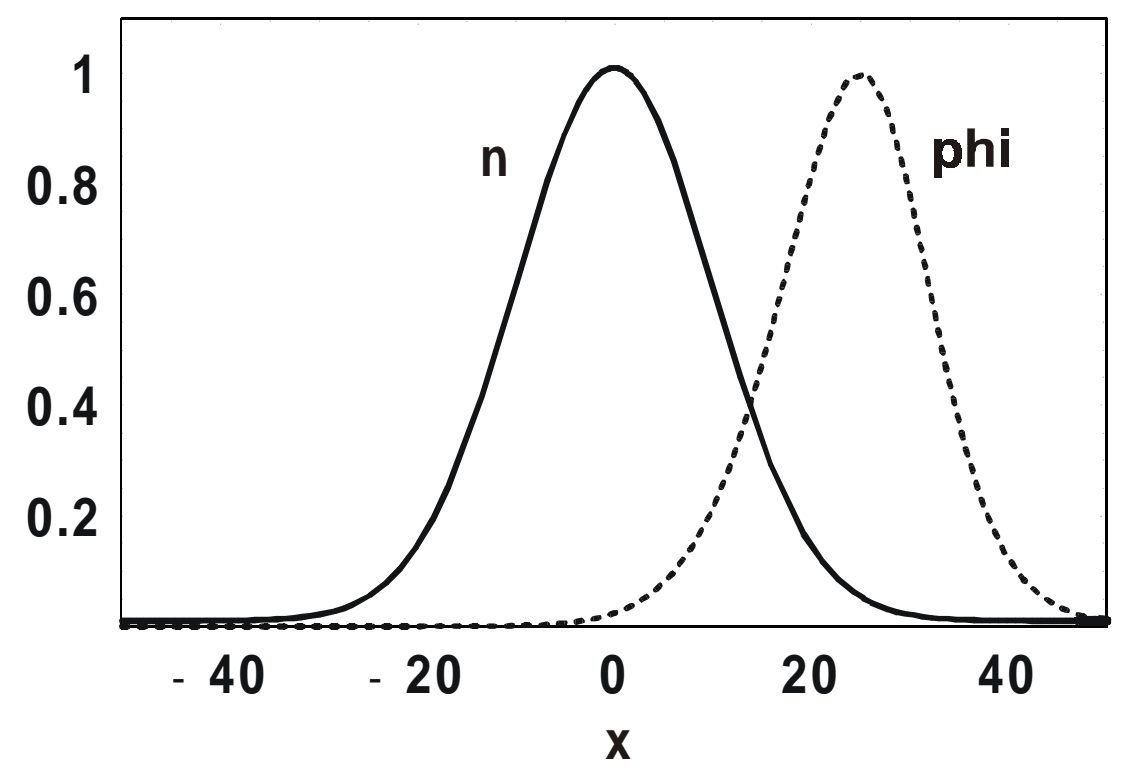

Fig. 1 Plots of the density profile $\overline{\mathrm{n}}(\mathrm{x})$ of the poloidally-elongated blob (solid curve, labeled "n") and the normalized eigenfunction $\tilde{\varphi}(x)$ of the most unstable mode (dashed curve, labeled "phi") for $\mathrm{k}_{\mathrm{y}}=0.25, \mathrm{x}_{\mathrm{b}}=10, \mathrm{D}=0.001, \mathrm{v}=0.1, \mathrm{q}=3$, and $\mathrm{n}_{\mathrm{f}}=0.01$.

Fig 1 


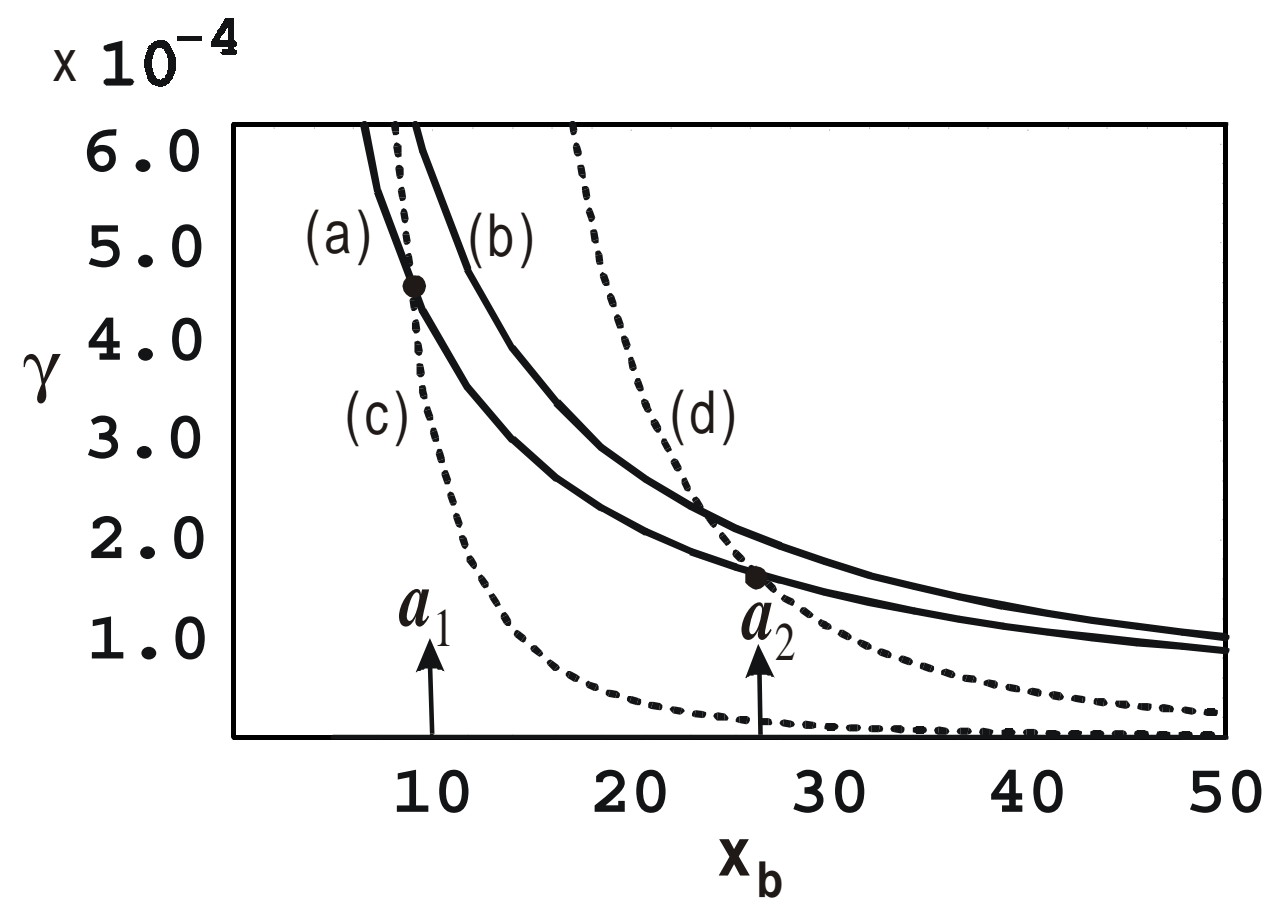

Fig. 2 Plots of the dimensionless instability growth rate and transport rates vs radial blob size $\mathrm{x}_{\mathrm{b}}$ for poloidally-elongated blobs. Here, $\gamma$ and $\mathrm{x}_{\mathrm{b}}$ are in units of $\Omega_{\mathrm{i}}$ and $\rho_{\mathrm{s}}$, respectively. Shown are (a) $\gamma_{\max }\left(x_{b}\right)$ for the non-local numerical solution (lower solid curve), (b) $\gamma_{\max }\left(x_{b}\right)$ for the local analytic solution (upper solid curve), (c) $\gamma_{c}\left(x_{b}\right) / 10$ (lower dashed curve) and (d) $\gamma_{c}\left(x_{b}\right)$ (upper dashed curve). The parameters are $\mathrm{D}=0.001, \mathrm{v}=0.1, \mathrm{q}=3$ and $\mathrm{n}_{\mathrm{f}}=0.01$. The dots and arrows indicate the crossings of the stability and transport curves at $\mathrm{x}_{\mathrm{b}}=a_{1}$ and $a_{2}$, as explained in the text.

Fig 2 


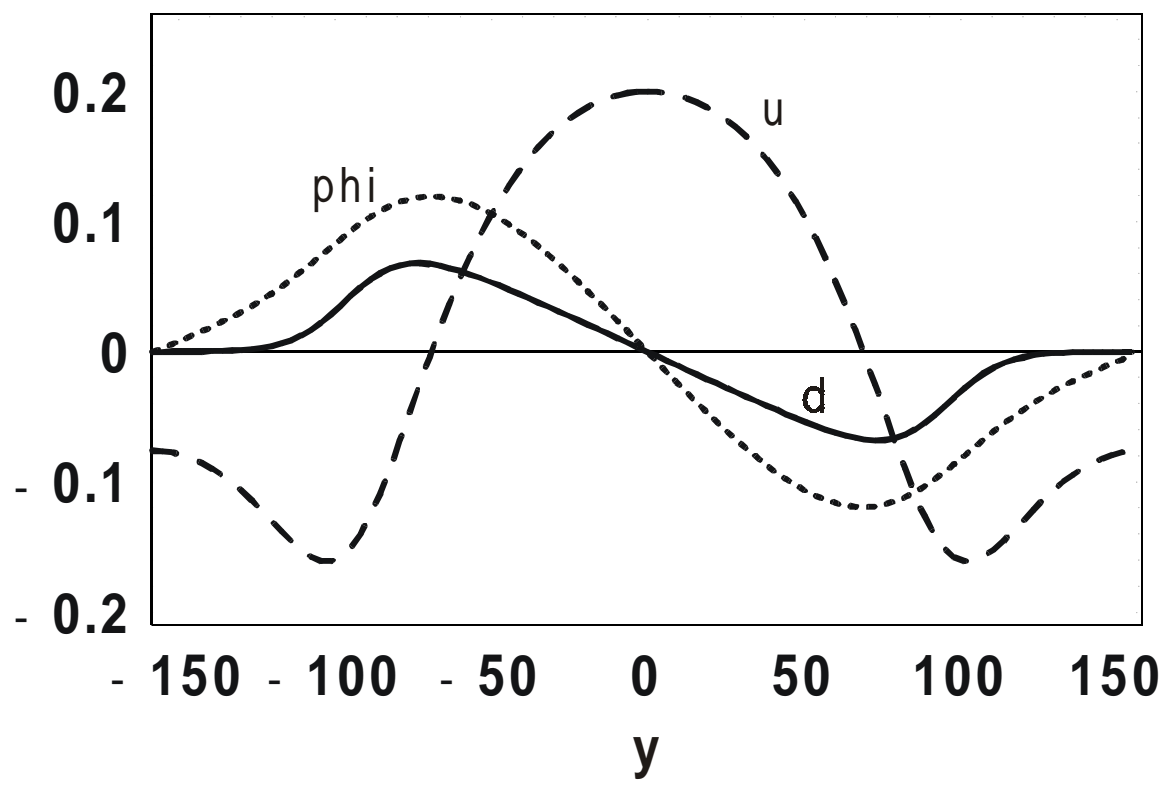

Fig. 3 Equilibrium profiles for the radially-elongated blob. Shown are the density weighting $\mathrm{d}(\mathrm{y})=\overline{\mathrm{n}}^{\prime}(\mathrm{y}) / \overline{\mathrm{n}}$ for the curvature drive (solid curve, labeled "d"), the equilibrium potential $\bar{\varphi}(\mathrm{y})$ (short-dashed curve, labeled "phi"), and the radial velocity $\mathrm{u}_{\mathrm{x}}(\mathrm{y})$ with arbitrary normalization (long-dashed curve, labeled "u") for the parameters $\mathrm{y}_{\mathrm{b}}=30, \mathrm{D}=0.001, \mathrm{v}=0.1, \mathrm{q}=3$, and $\mathrm{n}_{\mathrm{f}}=0.01$.

Fig 3 


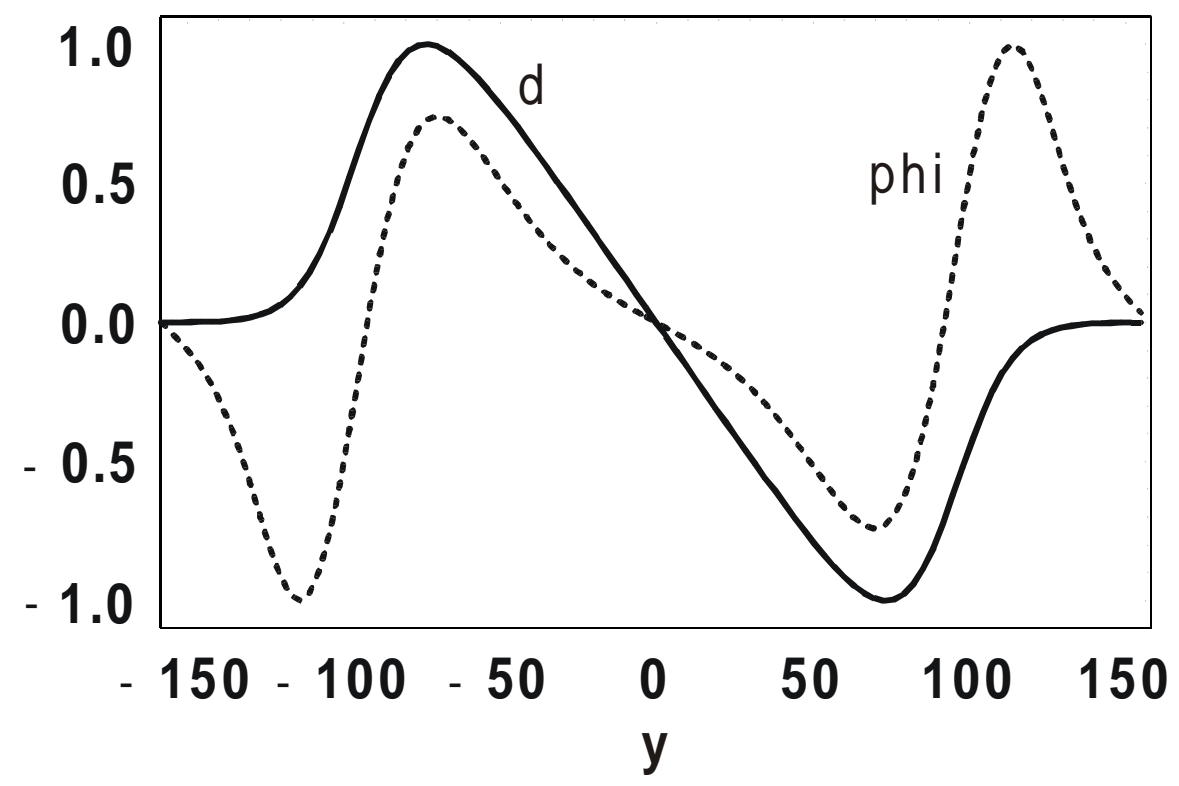

Fig. 4 Comparison of $d(y)=\bar{n}^{\prime}(y) / \bar{n}$ with arbitrary normalization (solid line, labeled "d") and the real part of the most unstable eigenfunction $\tilde{\varphi}(\mathrm{y})$ (dashed line, labeled "phi") for the radially-elongated blob with parameters $\mathrm{k}_{\mathrm{x}}=0.05, \mathrm{y}_{\mathrm{b}}=20$, $\mathrm{D}=0.001, v=0.1, \mathrm{q}=3$ and $\mathrm{n}_{\mathrm{f}}=0.01$.

Fig 4 


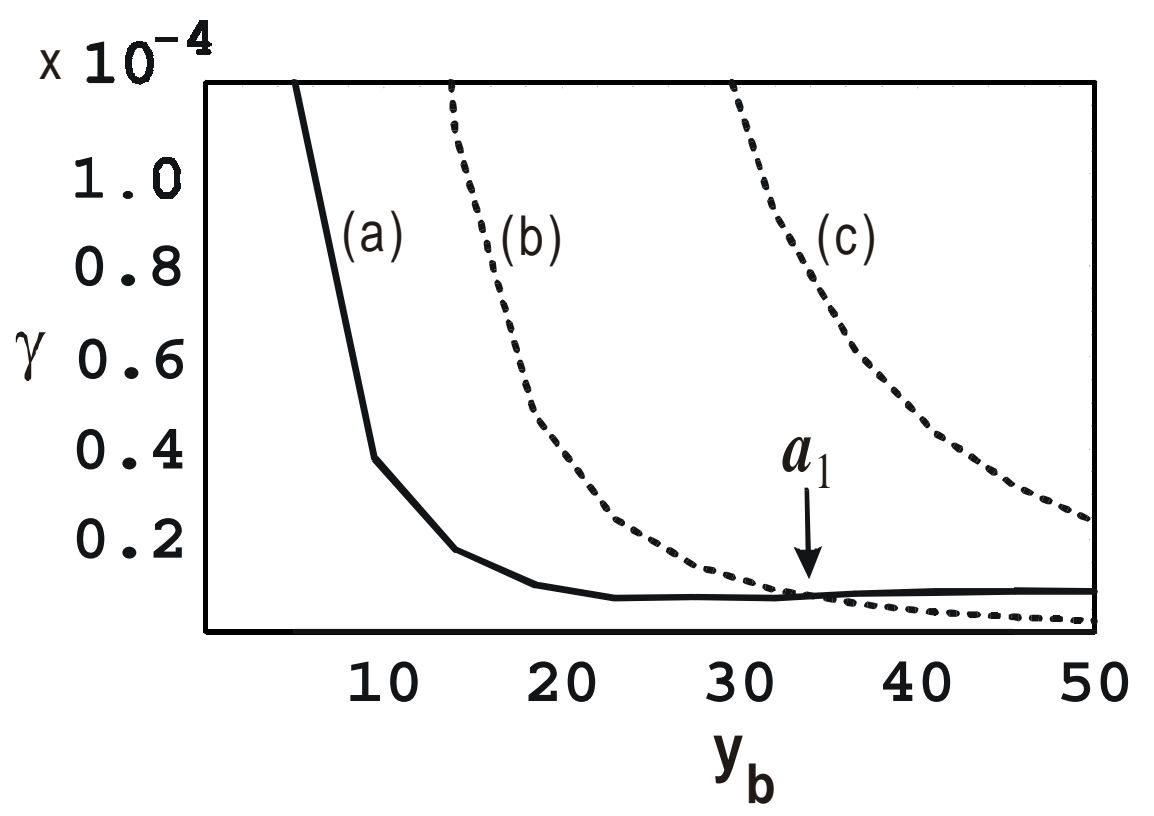

Fig. 5 Plots of the dimensionless instability growth rate and transport rates vs poloidal blob size $\mathrm{y}_{\mathrm{b}}$ for radially-elongated blobs. Here, $\gamma$ and $\mathrm{y}_{\mathrm{b}}$ are in units of $\Omega_{\mathrm{i}}$ and $\rho_{\mathrm{s}}$, respectively. Shown are (a) $\gamma_{\max }\left(y_{b}\right)$ for the non-local numerical solution (solid curve), (b) $\gamma_{c}\left(y_{b}\right) / 10$ (lower dashed curve), and (c) $\gamma_{c}\left(y_{b}\right)$ (upper dashed curve). The parameters are $\mathrm{D}=0.001, \mathrm{v}=0.1, \mathrm{q}=3$ and $\mathrm{n}_{\mathrm{f}}=0.01$. The arrow indicates the crossing of the stability and transport curves.

Fig 5 


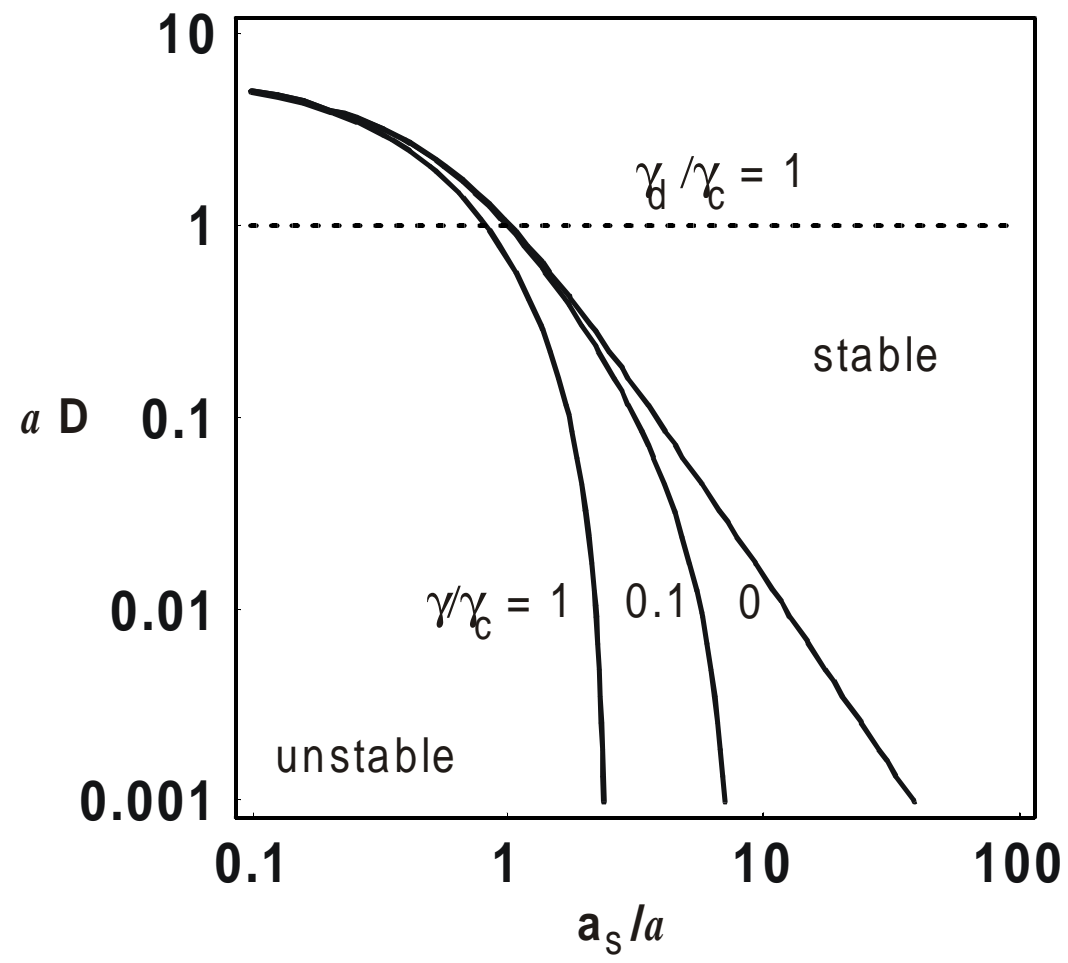

Fig. 6 Equilibrium, stability and transport boundaries in the $\left(\hat{a}_{s}, \hat{D}\right)$ plane for poloidallyelongated blobs with $\mathrm{q}=3$. The parameters $\hat{\mathrm{a}}_{\mathrm{s}}=\mathrm{a}_{\mathrm{s}} / a$ and $\hat{\mathrm{D}}=a \mathrm{D}$ scale the blob radius $a$ out of the stability problem.

Fig 6 


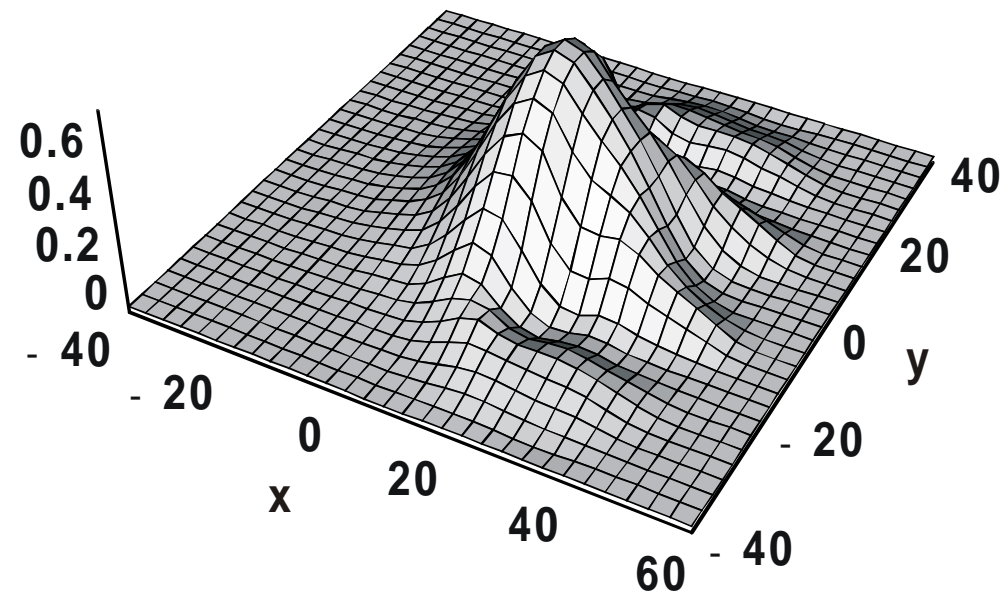

Fig. 7 Strongly unstable density blob in the nonlinear phase. The blob was initialized using Eq. (10) with $\mathrm{x}_{\mathrm{b}}=\mathrm{y}_{\mathrm{b}}=10, \mathrm{n}_{\mathrm{b}}=1$ and $\mathrm{n}_{\mathrm{f}}=0.01$; the other parameters are $\mathrm{D}$ $=0.01, \mathrm{a}_{\mathrm{s}}=10^{-4}$, and $\mathrm{t}=1.75 \tau_{\mathrm{c}}$.

Fig 7 

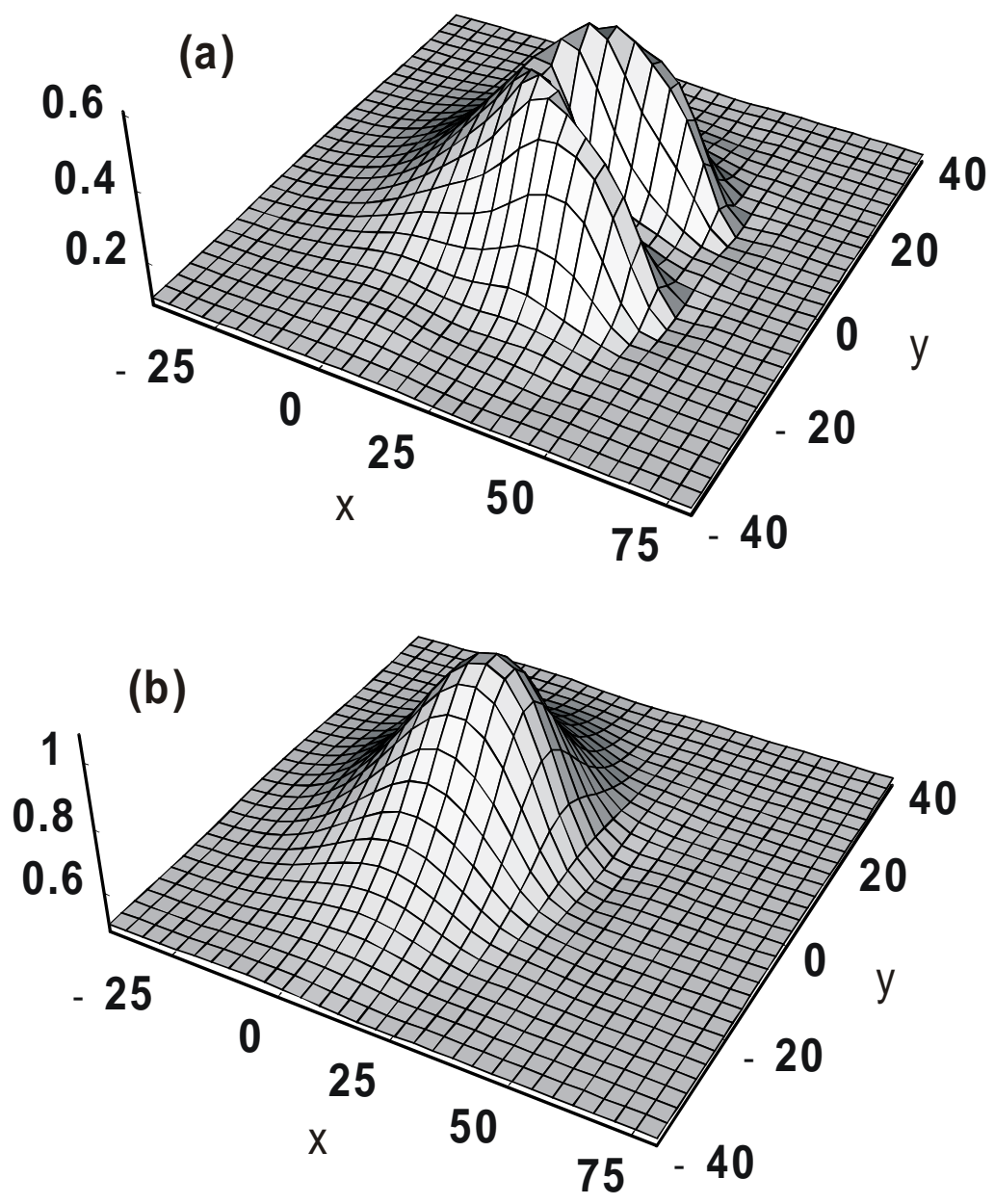

Fig. 8 Blob propagating on a floor density $\mathrm{n}_{\mathrm{f}}$ of (a) 0.1 and (b) 0.5 after the elapsed time $\mathrm{t}=3.8 \tau_{\mathrm{c}}$. The blob was initialized using Eq. (10) with $\mathrm{x}_{\mathrm{b}}=\mathrm{y}_{\mathrm{b}}=10$ and $\mathrm{n}_{\mathrm{b}}=1$; the other parameters are $\mathrm{D}=0.01$ and $\mathrm{a}_{\mathrm{s}}=0$. Case (a) is clearly in the nonlinear phase $(\gamma t>1)$ whereas case $(b)$ is not yet unstable.

Fig 8 

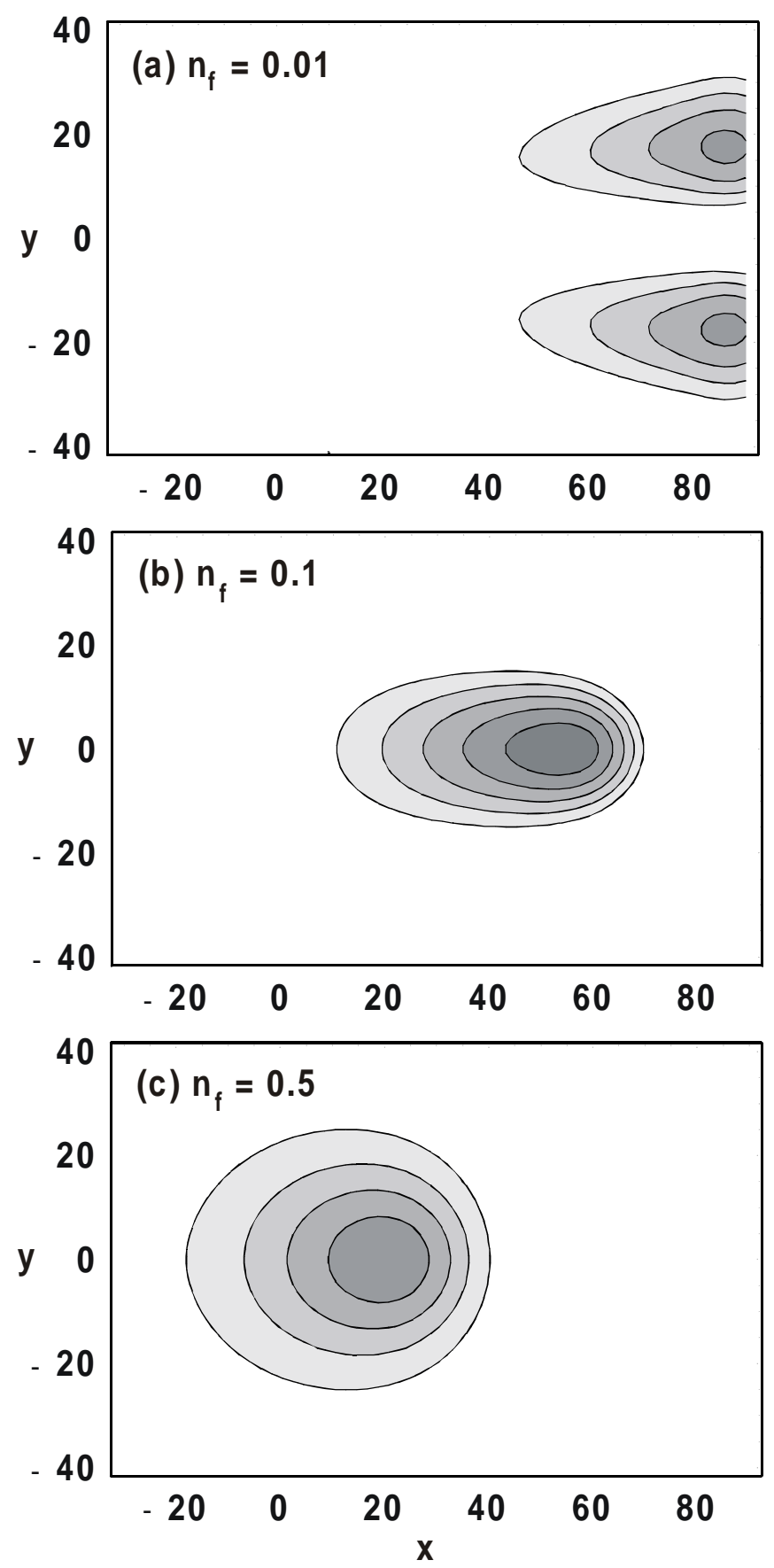

Fig. 9 Blob propagating on a floor density $\mathrm{n}_{\mathrm{f}}$ of (a) 0.01 , (b) 0.1 and (c) 0.5 for the parameters $\mathrm{D}=0.005$ and $\mathrm{a}_{\mathrm{s}}=7$. The blob was initialized using Eq. (10) with $\mathrm{x}_{\mathrm{b}}$ $=\mathrm{y}_{\mathrm{b}}=10$ and $\mathrm{n}_{\mathrm{b}}=1$. The elapsed time is $\mathrm{t}=12.5 \tau_{\mathrm{c}}$.

Fig 9 


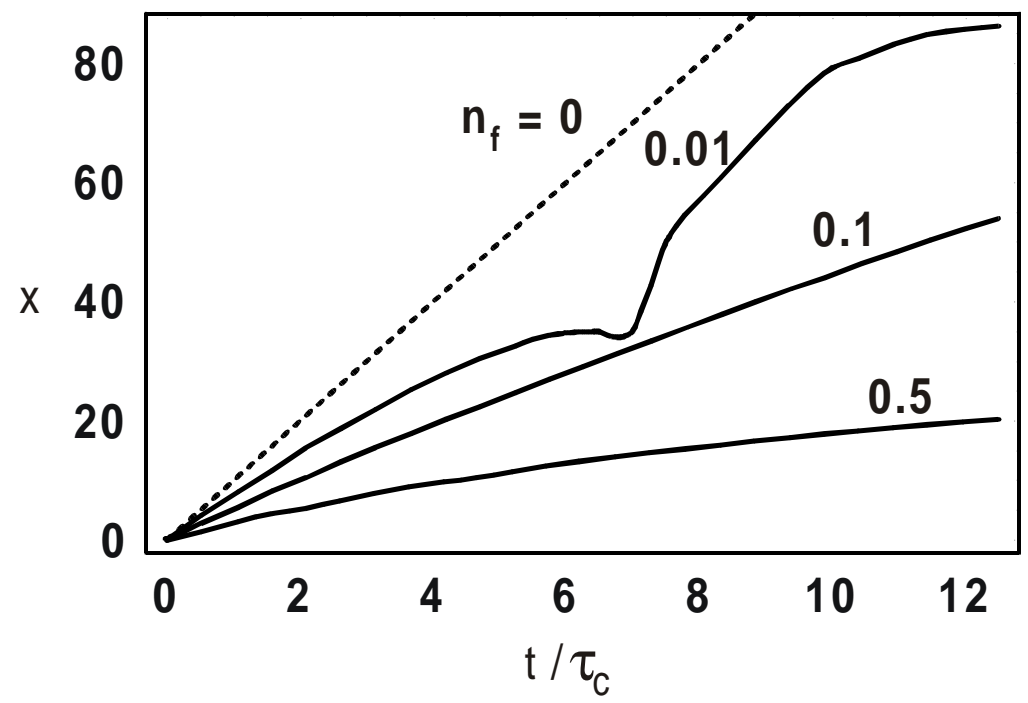

Fig. 10 Blob displacement $x$ vs $t / \tau_{c}$ for the analytic result in Eq. (1) with $D=a_{s}=n_{f}=0$ (dashed line), and the three cases shown in Fig. 9 (solid curves). The latter cases have a floor density $\mathrm{n}_{\mathrm{f}}$ of $0.01,0.1$, and 0.5 , respectively, with $\mathrm{D}=0.005$ and $\mathrm{a}_{\mathrm{s}}=$ 7. Note that the $\mathrm{n}_{\mathrm{f}}=0.01$ case exhibits slow-down before bifurcation and speedup afterwards. The reduced $u_{x}$ for the solid curves is due to both the effects of non-zero background density and viscosity.

Fig 10 

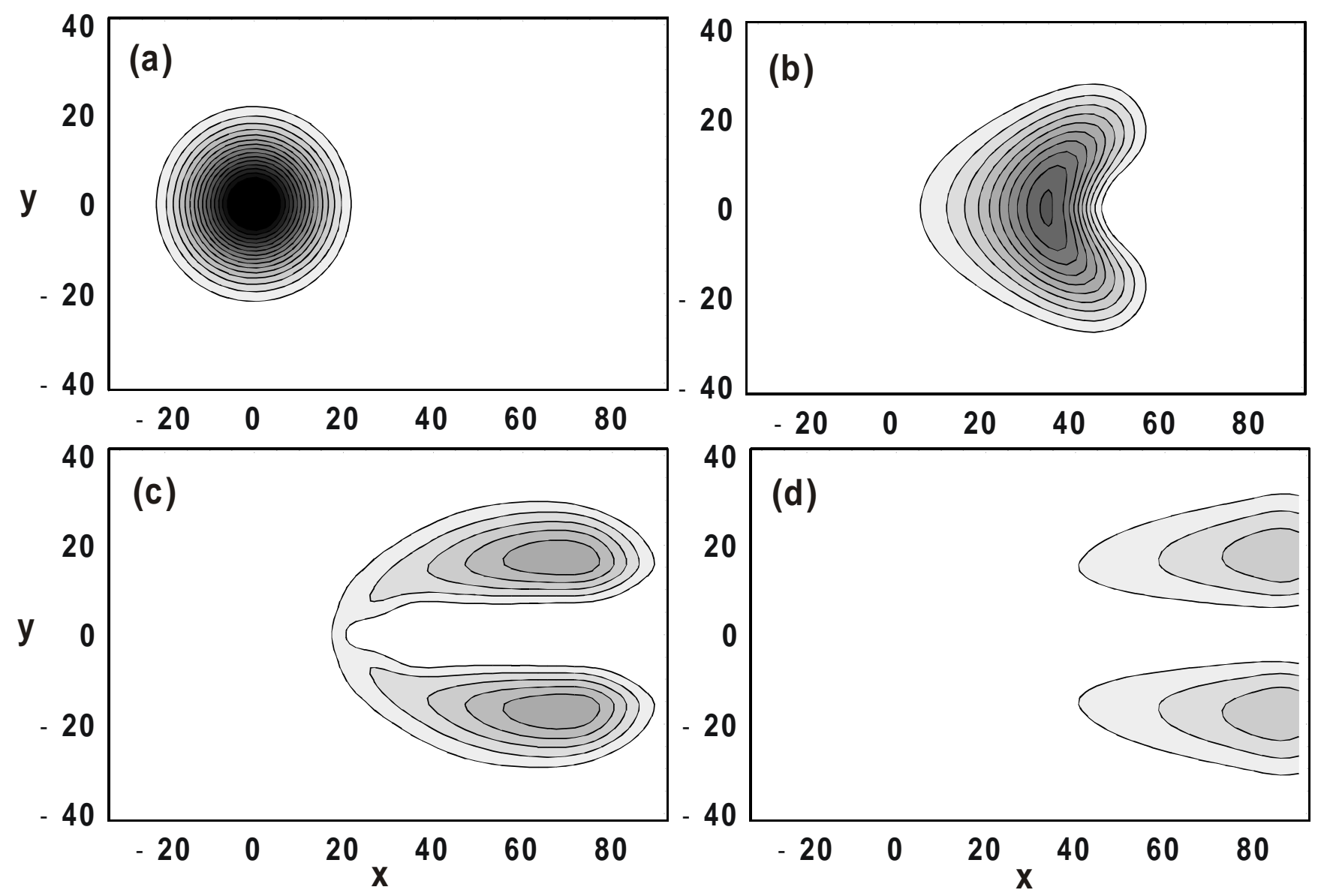

Fig. 11 Blob propagation with finite viscosity showing weak instability and subsequent bifurcation for the case $n_{f}=0.01$ in Fig. 10 at four values of $t / \tau_{c}$ : (a) 0 , (b) 6 , (c) 9 , and (d) 12 .

Fig 11 

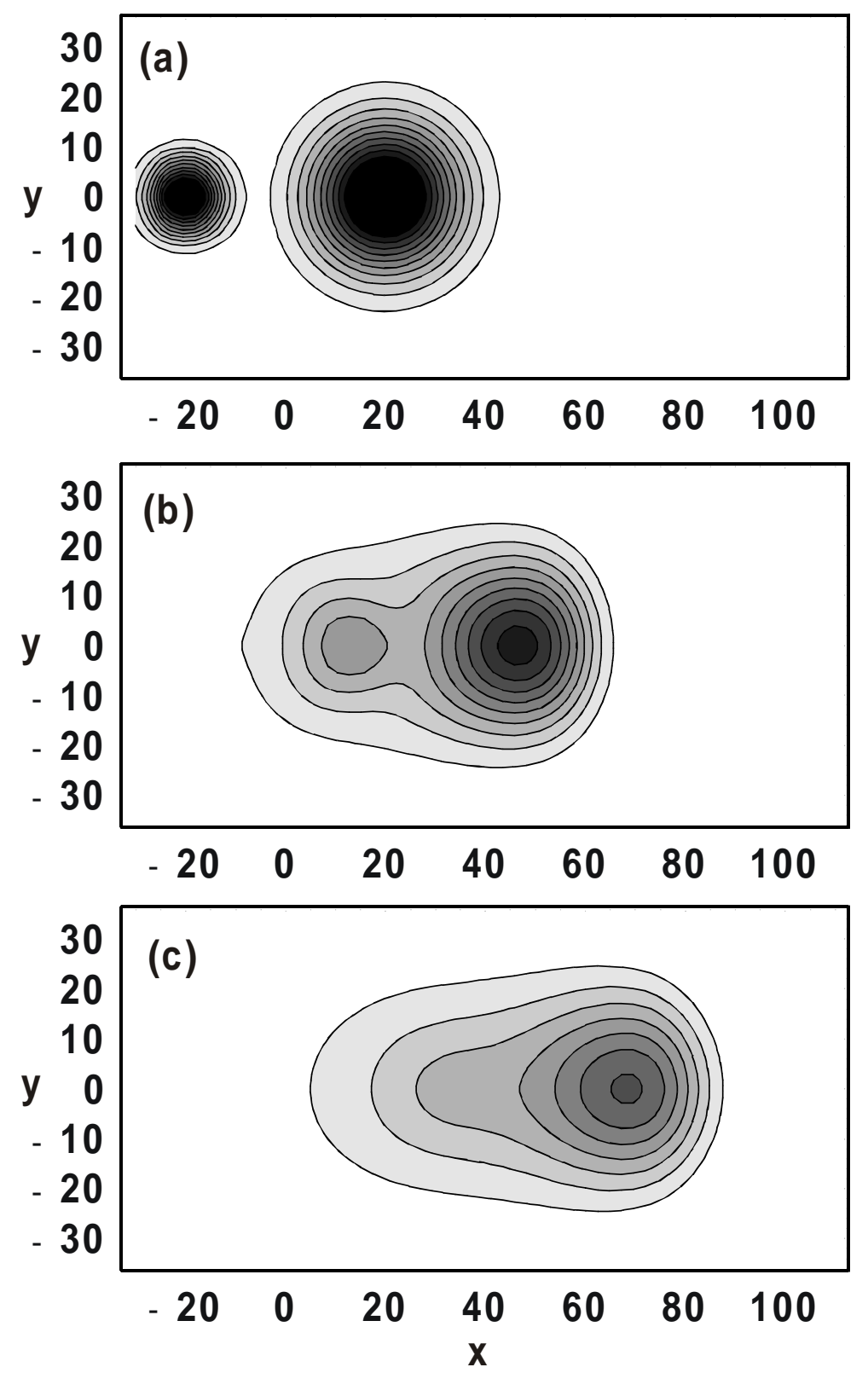

Fig. 12 Merger of a small and large blob for the parameters $D=0.005, a_{s}=10$ and $n_{f}=$ 0.01. The simulation results are shown at three values of $t / \tau_{c}$ : (a) 0 , (b) 4.5 , and (c) 9 .

Fig 12 